\title{
Infinite Horizon Stochastic Delay Evolution Equations in Hilbert Spaces and Stochastic Maximum Principle
}

\author{
Han Li, Jianjun Zhou*, Haoran Dai, Biteng Xu and Wenxu Dong
}

\begin{abstract}
In this paper, a class of infinite horizon optimal control problems is established, where the state equation is given by a stochastic delay evolution equation (SDEE), and the corresponding adjoint equation is given by an anticipated backward stochastic evolution equation (ABSEE). Firstly, we extend the form of Itô formula. After that, we establish the priori estimate for the solution to ABSEEs, and then the existence and uniqueness results of ABSEEs on infinite horizon are obtained. Finally, we establish necessary and sufficient conditions of stochastic maximum principle for infinite horizon optimal control problem in the form of Pontryagin's maximum principle.
\end{abstract}

\section{Introduction}

With the development of industrial technology and modern computer technology, we can widely observe the phenomenon of delay in many practical systems, such as in physics, biology, engineering, economics and other fields. The phenomenon of delay is that the system changes not only according to the current state, but also according to its past state. Stochastic delay differential equations (SDDEs) are designed to describe this phenomenon in these systems. Mohammed [18] proved the existence and uniqueness of the solution of SDDEs. Therefore, its theoretical reliability makes it possible to consider the stochastic optimal control problems of delay deeply. In the late years, the problem of optimal control with delay has been extensively studied. Readers can refer to the paper 4]. Moreover, stochastic evolution equations (SEEs) have been a hot topic in the study of dynamical systems. Da Prato and Zabczyk [6] systematically introduced the basic results of SEEs.

In the 1950s, with the development of aerospace technology and missile technology, an important branch of modern control theory has emerged: optimal control theory. The

Received August 9, 2020; Accepted December 2, 2021.

Communicated by Jein-Shan Chen.

2020 Mathematics Subject Classification. 93E20, 60H30, 49K27.

Key words and phrases. infinite horizon, anticipated backward stochastic evolution equations, stochastic maximum principle, optimal control.

This work was partially supported by the National Natural Science Foundation of China (Grant No. 11401474), the Natural Science Foundation of Shaanxi Province (Grant No. 2021JM-083) and the Fundamental Research Funds for the Central Universities (Grant Nos. 2452019075, 2452021063).

*Corresponding author. 
basic problem of optimal control theory is to seek a control for a controlled state system so that the performance index of the state system can reach the best. At present, optimal control theory has been widely used in social production and economic life, and plays an increasingly important role. Two basic methods to study optimal control problems are Bellman's dynamic programming principle and Pontryagin's maximum principle. Yong and Zhou [23] systematically illustrated the dynamic programming principle and the maximum principle method for stochastic optimal control. For Bellman's dynamic programming principle, the main idea is that the value function of the optimal control problem of the dynamic system satisfies its corresponding Hamilton-Jacobi-Bellman (HJB) equation. However, due to the infinite dimensional nature of delay systems, it is difficult to solve the optimal control problem with delay by using dynamic programming principle. Only in some special cases, the original infinite dimensional system can be simplified into some related finite dimensional systems [11]. But this method generally doesn't work. A typical approach to deal with optimal control problem with delay is to represent the control system in an appropriate infinite dimensional space without delay. For instance, under the sufficient condition of [12], we derive the equivalence between the original finite dimensional problem with delay and the infinite dimensional problem without delay in the sense of weak solution, then this problem can be solved by discussing the corresponding infinite dimensional HJB equations.

For Pontryagin's maximum principle, the main idea is to use the way of variation to solve the extremum problem of dynamic system. The research on the maximum principle can be roughly divided into two methods. One method includes a system involving threecoupled adjoint equations, and it consists of two backward stochastic differential equations (BSDEs) and one backward ordinary differential equation (ODE) [1,8, 19]. Different from the above papers, Shen, Meng and Shi 22] put forward a system that is composed of three-coupled BSDEs as adjoint equations with delay. In these papers, a hypothesis of the maximum principle is the third adjoint equation. In fact, this hypothesis is also to simplify the infinite dimensional control problem to the finite dimensional control problem. Therefore, the optimal conditions are established in this method only when the system with delay is finite dimensional in nature. The other method is that the adjoint equation is given by an anticipated backward stochastic differential equation (ABSDE) (see [21]). Chen and $\mathrm{Wu}$ [5] obtained the maximum principle for the controlled stochastic differential equations involving delays in both the state variable and the control variable. See also [2,16, 20.

This paper considers an optimal control problem for a class of infinite horizon stochastic evolution system with delay, and the system has pointwise delays in both state and control variables and no diffused delays. In fact, due to the randomness and time variability of operator $A$, if we define mild solutions as usual, the integrand in stochastic integral may 
not be adaptive. Thus, it seems more workable to study our optimal control problem in the weak solution framework of Gelfand triple $\left(V, H, V^{*}\right)$ (see 17$)$. In the meantime, our system is more general than [9]. The benefit of this framework is that a form of Itô formula is suitable for Gelfand triples $\left(V, H, V^{*}\right)$ in Hilbert spaces (see [15]). This Itô formula is of great significance to prove the existence and uniqueness theorem of solutions of stochastic delay evolution equations (SDEEs) and ABSEEs. Then, we consider optimal control problem for a class of infinite horizon controlled SDEEs in the Gelfand triple $\left(V, H, V^{*}\right)$ as follows:

$$
\begin{aligned}
x(t)= & \varphi(0)-\int_{0}^{t} A(s) x(s) d s+\int_{0}^{t} h(s, x(s), x(s-\delta), u(s), u(s-\delta)) d s \\
& +\int_{0}^{t} g(s, x(s), x(s-\delta), u(s), u(s-\delta)) d W(s), \quad t \in[0, \infty), \\
x(t)= & \varphi(t), \quad t \in[-\delta, 0], \\
u(t)= & u_{0}(t), \quad t \in[-\delta, 0],
\end{aligned}
$$

where the notations and mappings will be given in Sections 2 and 3 . Then, we define a class of optimal control problems on infinite horizon and the cost functional of the optimal control problems is given by

$$
J(u(\cdot))=\mathbb{E}\left[\int_{0}^{\infty} e^{-\lambda s} k(s, x(s), x(s-\delta), u(s), u(s-\delta)) d s\right],
$$

where $\lambda$ is a given attenuation coefficient and large enough, the control problem is understood in the usual weak sense (see [13]). Our aim is to find a control process $u(\cdot)$, within a set of admissible controls $(\bar{u}(\cdot) ; \bar{x}(\cdot))$, in such a way to minimize an infinite horizon cost functional

$$
J(\bar{u}(\cdot))=\inf _{u(\cdot) \in U_{\mathrm{ad}}} J(u(\cdot)) .
$$

And then, for any admissible pair $(u(\cdot) ; x(\cdot))$, we introduce the following adjoint equation in the Gelfand triple $\left(V, H, V^{*}\right)$

$$
d p(s)=\left\{A^{*} p(s)-\mathcal{H}_{x}(s)-\mathbb{E}\left[\mathcal{H}_{x_{\delta}}(s+\delta) \mid \mathcal{F}_{s}\right]\right\} d s+q(s) d W(s), \quad s \in[0, \infty),
$$

where we denote Hamiltonian by

$$
\begin{aligned}
\mathcal{H}\left(s, x, x_{\delta}, u, u_{\delta}, p, q\right):= & \left(h\left(s, x, x_{\delta}, u, u_{\delta}\right)-\lambda x, p\right)_{H} \\
& +\left(g\left(s, x, x_{\delta}, u, u_{\delta}\right), q\right)_{L_{2}(\Xi, H)}+k\left(s, x, x_{\delta}, u, u_{\delta}\right),
\end{aligned}
$$

and the notations will be given in Section 3. At the end, we deduce a variational formula of the cost functional from Hamiltonian. By using the variational formula, we obtain the 
necessary and sufficient conditions for the stochastic maximum principle of Pontryagin's type.

The present paper has the following improvements. We want to extend the results in [17] to infinite horizon case. This extension is nontrivial since it is difficult to extend ABSEEs from finite horizon to infinite horizon. Firstly, we give a priori estimate of the solution of ABSEEs on infinite horizon. Secondly, we obtain the existence and uniqueness theorem of solutions of linear BSEEs by using approximating methods. Finally, we establish the existence and uniqueness results of ABSEEs through the contraction mapping principle. And then we take the ABSEEs as the adjoint equation in our research of optimal control problems. We also note that our system is more general than $[7]$ in such a way that the unbounded operator $A$ in our control system is random and time-varying. In addition, we can obtain better result than $\left[7\right.$ if the operator $A$ satisfies $\langle A(s) x, x\rangle+\gamma\|x\|_{H}^{2} \geq \alpha\|x\|_{V}^{2}$, $\forall(s, x) \in[0, \infty) \times V$ for some constants $\alpha>0$ and $\gamma \in \mathbb{R}$. Specifically, Theorem 2.5 in this paper only needs $\beta<-(\gamma+19 L+1 / 2) \wedge 0$, while Theorem 2.4 in [7] requires $\beta<-132 L-1 / 2$. Here, $\beta \in \mathbb{R}$ is a constant such that $h(\cdot, 0,0) \in L_{\mathcal{P}}^{2}\left(\Omega ; L_{\beta}^{2}(H)\right)$ and $g(\cdot, 0,0) \in L_{\mathcal{P}}^{2}\left(\Omega ; L_{\beta}^{2}\left(L_{2}(\Xi, H)\right)\right)$, and $L^{1 / 2}$ denotes the Lipschitz coefficient of functions $h$ and $g$ with respect to the second and third variables.

The plan of this paper is organized as follows. In Section 2, we introduce some notations and spaces to establish our results, and then we extend the Itô formula into a new form in Hilbert space. After that, we establish the existence and uniqueness theorem of solutions of SDEEs and ABSEEs. In Section 3, we define a class of infinite horizon optimal control problem and establish necessary and sufficient conditions of stochastic maximum principle to optimal control problem. Finally, an example of the optimal control of a stochastic delay partial differential equation is presented in Section 4.

\section{Preliminaries}

\subsection{Notations and spaces}

Let $\Xi, H$ be a pair of real separable Hilbert spaces, for convenience, we shall use the notations $\|\cdot\|$ and $(\cdot, \cdot)$ for norms and scalar products with a subscript to specify the space, if necessary. $L(\Xi, H)$ denotes the space of all bounded linear operators from $\Xi$ into $H$, endowed with the usual operator norm. $L_{2}(\Xi, H)$ denotes the space of the Hilbert Schmidt operators from $\Xi$ into $H$, which is endowed with the Hilbert-Schmidt norm that makes it a separable Hilbert space.

Let $(\Omega, \mathcal{F}, P)$ be a complete probability space with a filtration $\left\{\mathcal{F}_{t}\right\}_{t \geq 0}$ which satisfies the usual condition, i.e., $\left\{\mathcal{F}_{t}\right\}_{t \geq 0}$ is a right continuous increasing family of sub $\sigma$-algebra of $\mathcal{F}$ and $\mathcal{F}_{0}$ contains all $P$-null sets of $\mathcal{F}$. By a cylindrical Wiener process with values in a 
Hilbert space $\Xi$, we mean a family $\{W(t), t \geq 0\}$ of linear mappings $\Xi \rightarrow L^{2}(\Omega)$, denoted $\xi \mapsto\left(\xi, W_{t}\right)$, such that for every $\xi, \eta \in \Xi,\{W(t) \xi, t \geq 0\}$ is a real Wiener process and $\mathbb{E}(W(t) \xi \cdot W(t) \eta)=(\xi, \eta)_{\Xi}$. The filtration $\left\{\mathcal{F}_{t}\right\}_{t \geq 0}$ is generated by cylindrical Wiener process $\{W(t), t \geq 0\}$. Let $\mathbb{E}$ denote the expectation under $P$. Let $\delta>0$ be a given constant and denote the time delay. Furthermore, we define $\mathcal{F}_{t} \equiv \mathcal{F}_{0}$ for all $t \in[-\delta, 0]$. All the concepts of measurability (e.g., predictability, independence, etc.) for stochastic processes refer to this filtration. By $\mathcal{P}$ we denote the predictable $\sigma$-algebra generated by predictable processes and by $\mathcal{B}(\Lambda)$ we denote, the Borel $\sigma$-algebra of any topological space $\Lambda$.

The following spaces will be frequently used in this paper:

- $L_{\mathcal{P}}^{p}\left(\Omega ; L_{\beta}^{q}(H)\right)$ : the space of processes $\{y(t), t \geq 0\}$, with values in $H$, defined for $\beta \in \mathbb{R}$ and $p, q \in[1, \infty)$. It is endowed with the norm

$$
\|y\|_{L_{\mathcal{P}}^{p}\left(\Omega ; L_{\beta}^{q}(H)\right)}^{p}=\mathbb{E}\left(\int_{0}^{\infty} e^{q \beta t}\|y(t)\|_{H}^{q} d t\right)^{p / q}
$$

is finite, and $y(t)$ admits a predictable version.

- $L_{\mathcal{P}}^{p}(\Omega ; C[0, T] ; H)$ : the space of predictable processes $\{y(t), t \in[0, T]\}$ with continuous paths in $H$, defined for $T>0$ and $p \in[1, \infty)$. It is endowed with the norm

$$
\|y\|_{L_{\mathcal{P}}^{p}(\Omega ; C[0, T] ; H)}^{p}=\mathbb{E} \sup _{t \in[0, T]}\|y(t)\|_{H}^{p}
$$

is finite.

- $L_{\mathcal{P}}^{q}\left(\Omega ; C_{\beta}(H)\right)$ : the space of predictable processes $\{y(t), t \geq 0\}$ with continuous paths in $H$, defined for $\beta \in \mathbb{R}$ and $q \in[1, \infty)$. It is endowed with the norm

$$
\|y\|_{L_{\mathcal{P}}^{q}\left(\Omega ; C_{\beta}(H)\right)}^{q}=\mathbb{E} \sup _{t \geq 0} e^{\beta q t}\|y(t)\|_{H}^{q}
$$

is finite.

- $L^{2}\left(\mathcal{F}_{t} ; H\right)$ : the space of measurable random variables $\xi$, with values in $H$. It is endowed with the norm

$$
\|\xi\|_{L^{2}\left(\mathcal{F}_{t} ; H\right)}^{2}=\mathbb{E}\|\xi\|_{H}^{2}
$$

is finite, for each $t \in[0, T]$.

With the time horizon $[0, T]$ replaced by $[-\delta, 0], L_{\mathcal{P}}^{p}(\Omega ; C[-\delta, 0] ; H)$ can be defined similarly as $L_{\mathcal{P}}^{p}(\Omega ; C[0, T] ; H)$. Let $H$ and $V$ denote two separable real Hilbert spaces such that $V$ is densely embedded in $H$. We identify $H$ with its dual space $H^{*}$ and denote 
by $V^{*}$ the dual of $V$. Then we have $V \subset H=H^{*} \subset V^{*}$. Denote by $(\cdot, \cdot)_{H}$ the inner product in $H$ and by $\langle\cdot, \cdot\rangle$ the duality product between $V$ and $V^{*}$.

Next, we provide the extended Itô formula for Hilbert-space-valued stochastic processes. Given processes $\{v(t, \omega),(t, \omega) \in[0, T] \times \Omega\},\{m(t, \omega),(t, \omega) \in[0, T] \times \Omega\}$, and $\left\{v^{*}(t, \omega),(t, \omega) \in[0, T] \times \Omega\right\}$ with values in $V, H, V^{*}$, respectively. Let $v(t, \omega)$ be measurable with respect to $(t, \omega)$ and be $\mathcal{F}_{t}$-measurable with respect to $\omega$ for a.e. $t$. For any $\eta \in V$, let the duality product $\left\langle\eta, v^{*}(t, \omega)\right\rangle$ is also $\mathcal{F}_{t}$-measurable with respect to $\omega$ for a.e. $t$. Suppose $m(t, \omega)$ is strongly continuous in $t, \mathcal{F}_{t}$-measurable with respect to $\omega$ for any $t$, and a local martingale with $m(0)=0$. Set $\langle m\rangle$ represent the increasing process portion for $\|m\|_{H}^{2}$ in the Doob-Meyer decomposition.

Lemma 2.1. Let $\phi \in L^{2}\left(\mathcal{F}_{0} ; H\right)$. Suppose that for any $\eta \in V$ and almost every $(t, \omega) \in$ $[0, T] \times \Omega$, the following equation is satisfied

$$
(\eta, v(t))_{H}=(\eta, \phi)_{H}+\int_{0}^{t}\left\langle\eta, v^{*}(s)\right\rangle d s+(\eta, m(t))_{H} .
$$

Then, there exists a set $\Omega^{\prime} \subset \Omega$ s.t. $P\left(\Omega^{\prime}\right)=1$ and an $H$-valued function $h(t)$ such that

(1) $h(t)$ is $\mathcal{F}_{t}$-measurable for any $t \in[0, T]$, and strongly continuous with respect to $t$ for any $\omega$, and $h(t)=v(t)$ for a.e. $(t, \omega) \in[0, T] \times \Omega$, and $h(0)=\phi$ for any $\omega \in \Omega^{\prime}$.

(2) for any $\omega \in \Omega^{\prime}, \beta \in \mathbb{R}$ and $t \in[0, T]$,

$$
\begin{aligned}
e^{2 \beta t}\|h(t)\|_{H}^{2}= & \|\phi\|_{H}^{2}+2 \beta \int_{0}^{t} e^{2 \beta s}\|h(s)\|_{H}^{2} d s+2 \int_{0}^{t} e^{2 \beta s}\left\langle v(s), v^{*}(s)\right\rangle d s \\
& +2 \int_{0}^{t} e^{2 \beta s}(h(s), d m(s))_{H}+\int_{0}^{t} e^{2 \beta s} d\langle m\rangle(s) .
\end{aligned}
$$

Proof. According to Theorem 3.2 in [15], we obtain the proof of (1) and energy equality

$$
\|h(t)\|_{H}^{2}=\|\phi\|_{H}^{2}+2 \int_{0}^{t}\left\langle v(s), v^{*}(s)\right\rangle d s+2 \int_{0}^{t}(h(s), d m(s))_{H}+\langle m\rangle(t) .
$$

Notice that the form of the right-side of the above formula, so $\|h(t)\|_{H}^{2}$ can be regarded as continuous semi-martingale. Therefore, we deduce the equation (2.1) by applying Itô formula for semi-martingale to $e^{2 \beta t}\|h(t)\|_{H}^{2}$.

\subsection{Forward stochastic delay evolution equation}

Now, let us consider a class of infinite horizon SDEE in the Gelfand triple $\left(V, H, V^{*}\right)$ as follows: 


$$
\begin{aligned}
x(t)= & \varphi(0)-\int_{0}^{t} A(s) x(s) d s+\int_{0}^{t} h(s, x(s), x(s-\delta)) d s \\
& +\int_{0}^{t} g(s, x(s), x(s-\delta)) d W(s), \quad t \in[0, \infty), \\
x(t)= & \varphi(t), \quad t \in[-\delta, 0],
\end{aligned}
$$

where $\{W(t), t \geq 0\}$ is a cylindrical Wiener process with values in a Hilbert space $\Xi$; the mapping $h:[0, \infty) \times \Omega \times H \times H \rightarrow H$ and $g:[0, \infty) \times \Omega \times H \times H \rightarrow L_{2}(\Xi, H)$ are $\mathcal{P} \otimes \mathcal{B}(H) \otimes \mathcal{B}(H) / \mathcal{B}(H)$-measurable and $\mathcal{P} \otimes \mathcal{B}(H) \otimes \mathcal{B}(H) / \mathcal{B}\left(L_{2}(\Xi, H)\right)$-measurable, respectively; the linear operator $A:[0, \infty) \times \Omega \rightarrow L\left(V, V^{*}\right)$ satisfies that $A v:[0, \infty) \times \Omega \rightarrow$ $V^{*}$ is $\mathcal{P} / \mathcal{B}\left(V^{*}\right)$-measurable for any $v \in V ; \varphi:[-\delta, 0] \times \Omega \rightarrow H$ is $\mathcal{P}_{[-\delta, 0]} / \mathcal{B}(H)$-measurable.

Moreover, we introduce the following assumptions:

Assumption 2.2. (i) For any $\left(x, x_{\delta}\right) \in H, h\left(\cdot, x, x_{\delta}\right), g\left(\cdot, x, x_{\delta}\right)$ are $\mathcal{F}$-progressively measurable. $h(\cdot, 0,0) \in L_{\mathcal{P}}^{2}\left(\Omega ; L_{\beta}^{2}(H)\right), g(\cdot, 0,0) \in L_{\mathcal{P}}^{2}\left(\Omega ; L_{\beta}^{2}\left(L_{2}(\Xi, H)\right)\right)$;

(ii) The operator A satisfies the following coercivity condition, i.e., there exist constants $\alpha>0$ and $\gamma \in \mathbb{R}$ such that

$$
\langle A(s) x, x\rangle+\gamma\|x\|_{H}^{2} \geq \alpha\|x\|_{V}^{2}, \quad \forall s \in[0, \infty), \forall x \in V
$$

(iii) The operator $A$ is uniformly bounded, i.e., there exists a constant $\bar{C}>0$ such that

$$
\sup _{(s, \omega) \in[0, \infty) \times \Omega}\|A(s, \omega)\|_{L\left(V, V^{*}\right)} \leq \bar{C}
$$

(iv) $h$ and $g$ are uniformly Lipschitz continuous in $\left(x, x_{\delta}\right)$, i.e., there exists a nonnegative constant $L$ such that for any $\left(x, x_{\delta}\right),\left(\bar{x}, \bar{x}_{\delta}\right) \in H \times H$ and a.e. $(s, \omega) \in[0, \infty) \times \Omega$,

$$
\begin{aligned}
& \left\|h\left(s, x, x_{\delta}\right)-h\left(s, \bar{x}, \bar{x}_{\delta}\right)\right\|_{H}^{2}+\left\|g\left(s, x, x_{\delta}\right)-g\left(s, \bar{x}, \bar{x}_{\delta}\right)\right\|_{L_{2}(\Xi, H)}^{2} \\
\leq & L\left(\|x-\bar{x}\|_{H}^{2}+\left\|x_{\delta}-\bar{x}_{\delta}\right\|_{H}^{2}\right) .
\end{aligned}
$$

Definition 2.3. An $H$-valued, $\mathbb{F}$-adapted process $x(\cdot)$ is called a solution to the SDEE $(2.2)$, if $x(\cdot) \in L_{\mathcal{P}}^{2}\left(\Omega ; L_{\beta}^{2}(H)\right) \cap L_{\mathcal{P}}^{2}\left(\Omega ; C_{\beta}(H)\right)$, such that for $\psi \in V$ and a.e. $(t, \omega) \in$ $[-\delta, \infty) \times \Omega$, it holds that

$$
\begin{aligned}
(x(t), \psi)_{H}= & (\varphi(0), \psi)_{H}-\int_{0}^{t}\langle A(s) x(s), \psi\rangle d s+\int_{0}^{t}(h(s, x(s), x(s-\delta)), \psi)_{H} d s \\
& +\int_{0}^{t}(g(s, x(s), x(s-\delta)), \psi)_{H} d W(s), \quad t \in[0, \infty), \\
x(t)= & \varphi(t), \quad t \in[-\delta, 0) .
\end{aligned}
$$


Theorem 2.4 (Existence and uniqueness theorem of finite horizon SDEE). Suppose that Assumption 2.2 holds, for every $T>0$, there exists a unique process $x(\cdot) \in L_{\mathcal{P}}^{2}(\Omega ; C[0, T]$; $H)$, a solution of the SDEE (2.2) on finite horizon $[0, T]$.

Proof. The proof of this theorem has been obtained in [3] and will not be repeated here.

Theorem 2.5 (Existence and uniqueness theorem of infinite horizon SDEE). Suppose that Assumption 2.2 holds for some $\beta \in \mathbb{R}$. Then for $\beta<-(\gamma+19 L+1 / 2) \wedge 0$, the infinite horizon SDEE (2.2) has a unique solution $x(\cdot) \in L_{\mathcal{P}}^{2}\left(\Omega ; L_{\beta}^{2}(H)\right) \cap L_{\mathcal{P}}^{2}\left(\Omega ; C_{\beta}(H)\right)$.

Proof. The existence and uniqueness of the solution of SDEE (2.2) can be obtained by Theorem 2.4. Now we prove $x(\cdot) \in L_{\mathcal{P}}^{2}\left(\Omega ; L_{\beta}^{2}(H)\right) \cap L_{\mathcal{P}}^{2}\left(\Omega ; C_{\beta}(H)\right)$. Applying Lemma 2.1 to $e^{2 \beta t}\|x(t)\|_{H}^{2}$, we have

$$
\begin{aligned}
& e^{2 \beta t}\|x(t)\|_{H}^{2}+2 \int_{0}^{t} e^{2 \beta s}\langle A(s) x(s), x(s)\rangle d s \\
= & \|\varphi(0)\|_{H}^{2}+2 \beta \int_{0}^{t} e^{2 \beta s}\|x(s)\|_{H}^{2} d s+2 \int_{0}^{t} e^{2 \beta s}(h(s, x(s), x(s-\delta)), x(s))_{H} d s \\
& +2 \int_{0}^{t} e^{2 \beta s}(g(s, x(s), x(s-\delta)), x(s))_{H} d W(s)+\int_{0}^{t} e^{2 \beta s}\|g(s, x(s), x(s-\delta))\|_{L_{2}(\Xi, H)}^{2} d s .
\end{aligned}
$$

Using Assumption 2.2 and the inequality $2(a, b)_{H} \leq \varepsilon\|a\|_{H}^{2}+\frac{1}{\varepsilon}\|b\|_{H}^{2}$ for all $\varepsilon>0$, we have

$$
\begin{aligned}
& e^{2 \beta t}\|x(t)\|_{H}^{2}+2 \alpha \int_{0}^{t} e^{2 \beta s}\|x(s)\|_{V}^{2} d s \\
\leq & \|\varphi(0)\|_{H}^{2}+(2 \beta+2 \gamma+3 L+\varepsilon+1) \int_{0}^{t} e^{2 \beta s}\|x(s)\|_{H}^{2} d s \\
& +2 \int_{0}^{t} e^{2 \beta s}(g(s, x(s), x(s-\delta)), x(s))_{H} d W(s)+3 L \int_{0}^{t} e^{2 \beta s}\|x(s-\delta)\|_{H}^{2} d s \\
& +\frac{1}{\varepsilon} \int_{0}^{t} e^{2 \beta s}\|h(s, 0,0)\|_{H}^{2} d s+2 \int_{0}^{t} e^{2 \beta s}\|g(s, 0,0)\|_{L_{2}(\Xi, H)}^{2} d s .
\end{aligned}
$$

Noting that

$$
\int_{0}^{t} e^{2 \beta s}\|x(s-\delta)\|_{H}^{2} d s \leq e^{2 \beta \delta} \int_{-\delta}^{0} e^{2 \beta s}\|\varphi(s)\|_{H}^{2} d s+e^{2 \beta \delta} \int_{0}^{t} e^{2 \beta s}\|x(s)\|_{H}^{2} d s,
$$

we have

$$
\begin{aligned}
& e^{2 \beta t}\|x(t)\|_{H}^{2}+2 \alpha \int_{0}^{t} e^{2 \beta s}\|x(s)\|_{V}^{2} d s \\
\leq & \|\varphi(0)\|_{H}^{2}+\left(2 \beta+2 \gamma+3 L+3 L e^{2 \beta \delta}+\varepsilon+1\right) \int_{0}^{t} e^{2 \beta s}\|x(s)\|_{H}^{2} d s \\
& +2 \int_{0}^{t} e^{2 \beta s}(g(s, x(s), x(s-\delta)), x(s))_{H} d W(s)+3 L e^{2 \beta \delta} \int_{-\delta}^{0} e^{2 \beta s}\|\varphi(s)\|_{H}^{2} d s \\
& +\frac{1}{\varepsilon} \int_{0}^{t} e^{2 \beta s}\|h(s, 0,0)\|_{H}^{2} d s+2 \int_{0}^{t} e^{2 \beta s}\|g(s, 0,0)\|_{L_{2}(\Xi, H)}^{2} d s .
\end{aligned}
$$


Using Burkholder-Davis-Gundy inequality, we derive that

$$
\begin{aligned}
& \frac{1}{2} E \sup _{s \geq 0} e^{2 \beta s}\|x(s)\|_{H}^{2}+2 \alpha E \int_{0}^{\infty} e^{2 \beta s}\|x(s)\|_{V}^{2} d s \\
\leq & \left(2 \beta+2 \gamma+19 L+19 L e^{2 \beta \delta}+\varepsilon+1\right) E \int_{0}^{\infty} e^{2 \beta s}\|x(s)\|_{H}^{2} d s \\
& +E\|\varphi(0)\|_{H}^{2}+19 L e^{2 \beta \delta} E \int_{-\delta}^{0} e^{2 \beta s}\|\varphi(s)\|_{H}^{2} d s \\
& +\frac{1}{\varepsilon} E \int_{0}^{\infty} e^{2 \beta s}\|h(s, 0,0)\|_{H}^{2} d s+18 E \int_{0}^{\infty} e^{2 \beta s}\|g(s, 0,0)\|_{L_{2}(\Xi, H)}^{2} d s .
\end{aligned}
$$

By Assumption 2.2(i), we have

$$
E \int_{0}^{\infty} e^{2 \beta s}\|g(s, 0,0)\|_{L_{2}(\Xi, H)}^{2} d s<\infty ; \quad E \int_{0}^{\infty} e^{2 \beta s}\|h(s, 0,0)\|_{H}^{2} d s<\infty
$$

for every $\beta<-(\gamma+19 L+1 / 2) \wedge 0$, we can find $\varepsilon>0$ small enough such that $\beta<$ $-(\gamma+19 L+\varepsilon / 2+1 / 2) \wedge 0$, then we have proved $x(\cdot) \in L_{\mathcal{P}}^{2}\left(\Omega ; L_{\beta}^{2}(H)\right) \cap L_{\mathcal{P}}^{2}\left(\Omega ; C_{\beta}(H)\right)$.

Remark 2.6. We note that our system is more general than 77 in such a way that the unbounded operator $A$ in our control system is random and time-varying. Moreover, our result is better than [7]. Specifically, the above theorem only needs $\beta<-(\gamma+19 L+1 / 2) \wedge 0$, while Theorem 2.4 in 7 requires $\beta<-132 L-1 / 2$.

Theorem 2.7 (Continuous dependence theorem of infinite horizon SDEE). Suppose that Assumption 2.2 holds for some $\beta \in \mathbb{R}$. If $x(\cdot)$ is the solution to the SDEE (2.2) corresponding to the generator $(A, h, g, \varphi)$, and if $\bar{x}(\cdot)$ is the solution to the SDEE (2.2) corresponding to the generator $(A, \bar{h}, \bar{g}, \bar{\varphi})$, then for $\beta<-(\gamma+19 L+1) \wedge 0$, the following estimate holds:

$$
\begin{aligned}
& \quad \frac{1}{2} E \sup _{s \geq 0}\left[e^{2 \beta s}\|x(s)-\bar{x}(s)\|_{H}^{2}\right]+2 \alpha E \int_{0}^{\infty} e^{2 \beta s}\|x(s)-\bar{x}(s)\|_{V}^{2} d s \\
& \leq\left(2 \beta+2 \gamma+19 L+19 L e^{2 \beta \delta}+2\right) E \int_{0}^{\infty} e^{2 \beta s}\|x(s)-\bar{x}(s)\|_{H}^{2} d s \\
& \quad+E\|\varphi(0)-\bar{\varphi}(0)\|_{H}^{2}+19 L e^{2 \beta \delta} E \int_{-\delta}^{0} e^{2 \beta s}\|\varphi(s)-\bar{\varphi}(s)\|_{H}^{2} d s \\
& +E \int_{0}^{\infty} e^{2 \beta s}\|h(s, \bar{x}(s), \bar{x}(s-\delta))-\bar{h}(s, \bar{x}(s), \bar{x}(s-\delta))\|_{H}^{2} d s \\
& +18 E \int_{0}^{\infty} e^{2 \beta s}\|g(s, \bar{x}(s), \bar{x}(s-\delta))-\bar{g}(s, \bar{x}(s), \bar{x}(s-\delta))\|_{L_{2}(\Xi, H)}^{2} d s .
\end{aligned}
$$

Proof. The proof of this theorem is similar to Theorem 2.5, and it will not be repeated here. 


\subsection{Anticipated backward stochastic evolution equation}

In this subsection, we will study the following ABSEE in the Gelfand triple $\left(V, H, V^{*}\right)$, which will be used in the next section. For its own research is also of great significance,

$$
\begin{aligned}
d y(s)= & \left\{A^{*}(s) y(s)+\mathbb{E}\left[\phi(s, y(s), y(s+\delta), z(s), z(s+\delta))+\theta y(s)-f(s) \mid \mathcal{F}_{s}\right]\right\} d s \\
& +z(s) d W(s), \quad s \in[0, \infty),
\end{aligned}
$$

where $\{W(t), t \geq 0\}$ is a cylindrical Wiener process with values in a Hilbert space $\Xi$; the mapping $\phi:[0, \infty) \times \Omega \times H \times H \times L_{2}(\Xi, H) \times L_{2}(\Xi, H) \rightarrow H$ is $\mathcal{P} \otimes \mathcal{B}(H) \otimes \mathcal{B}(H) \otimes$ $\mathcal{B}\left(L_{2}(\Xi, H)\right) \otimes \mathcal{B}\left(L_{2}(\Xi, H)\right) / \mathcal{B}(H)$-measurable; the mapping $f:[0, \infty) \times \Omega \rightarrow H$ is a predictable process with integrable paths; the mapping $z:[0, \infty) \times \Omega \rightarrow L_{2}(\Xi, H) ; \theta$ is a given real parameter; the operator $A^{*}$ is the adjoint operator of $A$, then by Remark 2.1 of [17], $A^{*}(s, \omega) \in L\left(V, V^{*}\right)$ for all $(s, \omega) \in[0, \infty) \times \Omega$, and by Proposition 1.3 on page 17 of [6], $A^{*} v:[0, \infty) \times \Omega \rightarrow V^{*}$ is $\mathcal{P} / \mathcal{B}\left(V^{*}\right)$-measurable for any $v \in V$.

Moreover, we introduce the following assumptions:

Assumption 2.8. (i) There exist $\mu, \beta \in \mathbb{R}$, such that for all $s \in[0, \infty), y, \bar{y}, y_{\delta}, \bar{y}_{\delta} \in H$ and $z, z_{\delta} \in L_{2}(\Xi, H)$,

$$
\begin{gathered}
\left(\phi\left(s, y, y_{\delta}, z, z_{\delta}\right)-\phi\left(s, \bar{y}, \bar{y}_{\delta}, z, z_{\delta}\right), y-\bar{y}\right)_{H} \geq \mu\left(\|y-\bar{y}\|_{H}^{2}+\left\|y_{\delta}-\bar{y}_{\delta}\right\|_{H}^{2}\right), \\
\mathbb{E} \int_{0}^{\infty} e^{2 \beta s}\|\phi(s, 0,0,0,0)\|_{H}^{2} d s<\infty
\end{gathered}
$$

(ii) $\phi$ is Lipschitz continuous with respect to $\left(y, y_{\delta}, z, z_{\delta}\right)$, i.e., there exists a nonnegative constant $L$ such that for any $\left(y, y_{\delta}, z, z_{\delta}\right),\left(\bar{y}, \bar{y}_{\delta}, \bar{z}, \bar{z}_{\delta}\right) \in H \times H \times L_{2}(\Xi, H) \times L_{2}(\Xi, H)$ and a.e. $(s, \omega) \in[0, \infty) \times \Omega$,

$$
\begin{aligned}
& \left\|\phi\left(s, y, y_{\delta}, z, z_{\delta}\right)-\phi\left(s, \bar{y}, \bar{y}_{\delta}, \bar{z}, \bar{z}_{\delta}\right)\right\|_{H} \\
\leq & L\left(\|y-\bar{y}\|_{H}+\left\|y_{\delta}-\bar{y}_{\delta}\right\|_{H}+\|z-\bar{z}\|_{L_{2}(\Xi, H)}+\left\|z_{\delta}-\bar{z}_{\delta}\right\|_{L_{2}(\Xi, H)}\right) .
\end{aligned}
$$

We note that from the properties of the adjoint operator, if $A$ satisfies (ii) and (iii) in Assumption 2.2, its adjoint operator $A^{*}$ naturally satisfies (ii) and (iii) in Assumption 2.2 (see Remark 2.2 in 17$]$ ).

Definition 2.9. An $\left(H \times L_{2}(\Xi, H)\right)$-valued, $\mathbb{F}$-adapted process $(y(\cdot), z(\cdot))$ is called a solution to the ABSEE (2.4), if $(y(\cdot), z(\cdot)) \in L_{\mathcal{P}}^{2}\left(\Omega ; L_{\beta}^{2}(H)\right) \times L_{\mathcal{P}}^{2}\left(\Omega ; L_{\beta}^{2}\left(L_{2}(\Xi, H)\right)\right)$ and $f(\cdot) \in L_{\mathcal{P}}^{2}\left(\Omega ; L_{\beta}^{2}(H)\right)$ such that for $\psi \in V$ and a.e. $(t, T, \omega) \in[0, T] \times[0, \infty) \times \Omega$, for every $T>0$, it holds that

$$
(y(t), \psi)_{H}=(y(T), \psi)_{H}
$$




$$
\begin{aligned}
& -\int_{t}^{T}\left(\left\{\mathbb{E}\left[\phi(s, y(s), y(s+\delta), z(s), z(s+\delta))+\theta y(s)-f(s) \mid \mathcal{F}_{s}\right]\right\}, \psi\right)_{H} d s \\
& -\int_{t}^{T}\left\langle A^{*}(s) y(s), \psi\right\rangle d s-\int_{t}^{T}(z(s), \psi)_{H} d W(s), \quad 0 \leq t<T<\infty .
\end{aligned}
$$

Theorem 2.10. Suppose that Assumption 2.2(ii), (iii) and Assumption 2.8 hold for some $\beta \in \mathbb{R}, \theta \in \mathbb{R}$. If $(y(\cdot), z(\cdot))$ is the solution to the following ABSEE 2.5 corresponding to the generator $\left(A^{*}, \phi, f\right)$ :

$$
\begin{aligned}
y(T)-y(t)= & \int_{t}^{T} \mathbb{E}\left[\phi(s, y(s), y(s+\delta), z(s), z(s+\delta))+\theta y(s)-f(s) \mid \mathcal{F}_{s}\right] d s \\
& +\int_{t}^{T} A^{*}(s) y(s) d s+\int_{t}^{T} z(s) d W(s), \quad 0 \leq t \leq T<\infty .
\end{aligned}
$$

Suppose that $(\bar{y}(\cdot), \bar{z}(\cdot))$ is the solution to the ABSEE (2.5) corresponding to another generator $\left(A^{*}, \phi, \bar{f}\right)$. Then for every $\bar{\theta}>-\left(\beta+\mu-\gamma-\frac{L^{2}\left(1+e^{2 \beta \delta}\right)}{e^{2 \beta \delta}}+\frac{\mu}{e^{2 \beta \delta}}\right)$, and $\theta>\bar{\theta}$, there exists a constant $C_{1}>0$ such that

$$
\begin{aligned}
& (\theta-\bar{\theta})^{1 / 2}\|z(s)-\bar{z}(s)\|_{L_{\mathcal{P}}^{2}\left(\Omega ; L_{\beta}^{2}\left(L_{2}(\Xi, H)\right)\right)}+(\theta-\bar{\theta})\|y(s)-\bar{y}(s)\|_{L_{\mathcal{P}}^{2}\left(\Omega ; L_{\beta}^{2}(H)\right)} \\
& +(\theta-\bar{\theta})^{1 / 2} \sup _{s \geq 0} e^{2 \beta s} \mathbb{E}\|y(s)-\bar{y}(s)\|_{H}^{2}+(\theta-\bar{\theta})^{1 / 2}\|y(s)-\bar{y}(s)\|_{L_{\mathcal{P}}^{2}\left(\Omega ; L_{\beta}^{2}(V)\right)} \\
\leq & C_{1}\|f(s)-\bar{f}(s)\|_{L_{\mathcal{P}}^{2}\left(\Omega ; L_{\beta}^{2}(H)\right)},
\end{aligned}
$$

where $C_{1}$ depends only on $\beta, \mu, \gamma, L, \alpha$ and $\bar{\theta}$.

Proof. To simplify our notation, we write

$$
\begin{gathered}
\widehat{y}(s):=y(s)-\bar{y}(s), \quad \widehat{f}(s):=f(s)-\bar{f}(s), \quad \widehat{z}(s):=z(s)-\bar{z}(s), \\
\widehat{\phi}(s)=\phi(s, y(s), y(s+\delta), z(s), z(s+\delta))-\phi(s, \bar{y}(s), \bar{y}(s+\delta), \bar{z}(s), \bar{z}(s+\delta)) .
\end{gathered}
$$

According to (2.1), we obtain

$$
\begin{aligned}
& e^{2 \beta t}\|\widehat{y}(t)\|_{H}^{2}-e^{2 \beta T}\|\widehat{y}(T)\|_{H}^{2}+\int_{t}^{T} e^{2 \beta s}\left(2(\beta+\theta)\|\widehat{y}(s)\|_{H}^{2}+\|\widehat{z}(s)\|_{L_{2}(\Xi, H)}^{2}\right) d s \\
= & -2 \int_{t}^{T} e^{2 \beta s}\left(\widehat{y}(s), \mathbb{E}\left[\widehat{\phi}(s) \mid \mathcal{F}_{s}\right]\right)_{H} d s-2 \int_{t}^{T} e^{2 \beta s}\left\langle\widehat{y}(s), A^{*}(s) \widehat{y}(s)\right\rangle d s \\
& +2 \int_{t}^{T} e^{2 \beta s}(\widehat{y}(s), \widehat{f}(s))_{H} d s-2 \int_{t}^{T} e^{2 \beta s}(\widehat{y}(s), \widehat{z}(s) d W(s))_{H} .
\end{aligned}
$$

By Assumption 2.8(iii)(iv) and elementary inequality, we obtain

$$
\begin{aligned}
2\left(\widehat{y}(s), \mathbb{E}\left[\widehat{\phi}(s) \mid \mathcal{F}_{s}\right]\right)_{H} \geq & 2 \mu\left(\|\widehat{y}(s)\|_{H}^{2}+\mathbb{E}\left[\|\widehat{y}(s+\delta)\|_{H}^{2} \mid \mathcal{F}_{s}\right]\right) \\
& -2 L\|\widehat{y}(s)\|_{H}\left(\mathbb{E}\left[\|\widehat{z}(s+\delta)\|_{L_{2}(\Xi, H)} \mid \mathcal{F}_{s}\right]+\|\widehat{z}(s)\|_{L_{2}(\Xi, H)}\right) \\
\geq & \left(2 \mu-\frac{2 L^{2}}{\rho}\right)\|\widehat{y}(s)\|_{H}^{2}+2 \mu \mathbb{E}\left[\|\widehat{y}(s+\delta)\|_{H}^{2} \mid \mathcal{F}_{s}\right] \\
& -\rho\left(\mathbb{E}\left[\|\widehat{z}(s+\delta)\|_{L_{2}(\Xi, H)}^{2} \mid \mathcal{F}_{s}\right]+\|\widehat{z}(s)\|_{L_{2}(\Xi, H)}^{2}\right),
\end{aligned}
$$


where $\rho$ is an arbitrary number in $\left(0, \frac{e^{2 \beta \delta}}{1+e^{2 \beta \delta}}\right]$. Moreover, by Assumption 2.2(ii),

$$
-2 \int_{t}^{T} e^{2 \beta s}\left\langle\widehat{y}(s), A^{*}(s) \widehat{y}(s)\right\rangle d s \leq 2 \int_{t}^{T} e^{2 \beta s}\left[\gamma\|\widehat{y}(s)\|_{H}^{2}-\alpha\|\widehat{y}(s)\|_{V}^{2}\right] d s .
$$

Combining with the above inequality, by (2.6), we obtain

$$
\begin{aligned}
& e^{2 \beta t}\|\widehat{y}(t)\|_{H}^{2}-e^{2 \beta T}\|\widehat{y}(T)\|_{H}^{2}+\left(2 \beta+2 \theta+2 \mu-2 \gamma-\frac{2 L^{2}}{\rho}\right) \int_{t}^{T} e^{2 \beta s}\|\widehat{y}(s)\|_{H}^{2} d s \\
& +(1-\rho) \int_{t}^{T} e^{2 \beta s}\|\widehat{z}(s)\|_{L_{2}(\Xi, H)}^{2} d s+2 \alpha \int_{t}^{T} e^{2 \beta s}\|\widehat{y}(s)\|_{V}^{2} d s \\
& \quad-\rho \int_{t}^{T} e^{2 \beta s} \mathbb{E}\left[\|\widehat{z}(s+\delta)\|_{L_{2}(\Xi, H)}^{2} \mid \mathcal{F}_{s}\right] d s+2 \mu \int_{t}^{T} e^{2 \beta s} \mathbb{E}\left[\|\widehat{y}(s+\delta)\|_{H}^{2} \mid \mathcal{F}_{s}\right] d s \\
& \leq 2 \int_{t}^{T} e^{2 \beta s}(\widehat{y}(s), \widehat{f}(s))_{H} d s-2 \int_{t}^{T} e^{2 \beta s}(\widehat{y}(s), \widehat{z}(s) d W(s))_{H} .
\end{aligned}
$$

Noting that

$$
\begin{aligned}
& \rho \int_{t}^{T} e^{2 \beta s} \mathbb{E}\left[\|\widehat{z}(s+\delta)\|_{L_{2}(\Xi, H)}^{2} \mid \mathcal{F}_{s}\right] d s \\
= & \rho \int_{t+\delta}^{T+\delta} e^{2 \beta(l-\delta)} \mathbb{E}\left[\|\widehat{z}(l)\|_{L_{2}(\Xi, H)}^{2} \mid \mathcal{F}_{l-\delta}\right] d l \\
= & \frac{\rho}{e^{2 \beta \delta}} \int_{t+\delta}^{T} e^{2 \beta l} \mathbb{E}\left[\|\widehat{z}(l)\|_{L_{2}(\Xi, H)}^{2} \mid \mathcal{F}_{l-\delta}\right] d l+\frac{\rho}{e^{2 \beta \delta}} \int_{T}^{T+\delta} e^{2 \beta l} \mathbb{E}\left[\|\widehat{z}(l)\|_{L_{2}(\Xi, H)}^{2} \mid \mathcal{F}_{l-\delta}\right] d l
\end{aligned}
$$

in the same way, we obtain

$$
\begin{aligned}
& 2 \mu \int_{t}^{T} e^{2 \beta s} \mathbb{E}\left[\|\widehat{y}(s+\delta)\|_{H}^{2} \mid \mathcal{F}_{s}\right] d s \\
= & \frac{2 \mu}{e^{2 \beta \delta}} \int_{t+\delta}^{T} e^{2 \beta l} \mathbb{E}\left[\|\widehat{y}(l)\|_{H}^{2} \mid \mathcal{F}_{l-\delta}\right] d l+\frac{2 \mu}{e^{2 \beta \delta}} \int_{T}^{T+\delta} e^{2 \beta l} \mathbb{E}\left[\|\widehat{y}(l)\|_{H}^{2} \mid \mathcal{F}_{l-\delta}\right] d l .
\end{aligned}
$$

Then by the previous equation, we obtain

$$
\begin{aligned}
& e^{2 \beta t}\|\widehat{y}(t)\|_{H}^{2}-e^{2 \beta T}\|\widehat{y}(T)\|_{H}^{2}+2 \alpha \int_{t}^{T} e^{2 \beta s}\|\widehat{y}(s)\|_{V}^{2} d s \\
& +\left(2 \beta+2 \theta+2 \mu-2 \gamma-\frac{2 L^{2}}{\rho}\right) \int_{t}^{T} e^{2 \beta s}\|\widehat{y}(s)\|_{H}^{2} d s+(1-\rho) \int_{t}^{T} e^{2 \beta s}\|\widehat{z}(s)\|_{L_{2}(\Xi, H)}^{2} d s \\
& -\frac{\rho}{e^{2 \beta \delta}} \int_{t+\delta}^{T} e^{2 \beta s} \mathbb{E}\left[\|\widehat{z}(s)\|_{L_{2}(\Xi, H)}^{2} \mid \mathcal{F}_{s-\delta}\right] d s+\frac{2 \mu}{e^{2 \beta \delta}} \int_{t+\delta}^{T} e^{2 \beta s} \mathbb{E}\left[\|\widehat{y}(s)\|_{H}^{2} \mid \mathcal{F}_{s-\delta}\right] d s \\
& \leq-2 \int_{t}^{T} e^{2 \beta s}(\widehat{y}(s), \widehat{z}(s) d W(s))_{H}+2 \int_{t}^{T} e^{2 \beta s}(\widehat{y}(s), \widehat{f}(s))_{H} d s \\
& +\frac{\rho}{e^{2 \beta \delta}} \int_{T}^{T+\delta} e^{2 \beta s} \mathbb{E}\left[\|\widehat{z}(s)\|_{L_{2}(\Xi, H)}^{2} \mid \mathcal{F}_{s-\delta}\right] d s-\frac{2 \mu}{e^{2 \beta \delta}} \int_{T}^{T+\delta} e^{2 \beta s} \mathbb{E}\left[\|\widehat{y}(s)\|_{H}^{2} \mid \mathcal{F}_{s-\delta}\right] d s .
\end{aligned}
$$


For any $\varepsilon>0$, by inequality $2(a, b)_{H} \leq \varepsilon\|a\|_{H}^{2}+\frac{1}{\varepsilon}\|b\|_{H}^{2}$, we obtain

$$
2 \int_{t}^{T} e^{2 \beta s}(\widehat{y}(s), \widehat{f}(s))_{H} d s \leq \varepsilon \int_{t}^{T} e^{2 \beta s}\|\widehat{y}(s)\|_{H}^{2} d s+\frac{1}{\varepsilon} \int_{t}^{T} e^{2 \beta s}\|\widehat{f}(s)\|_{H}^{2} d s .
$$

Using the quadratic variation of stochastic integrals to (2.7), we obtain

$$
\begin{aligned}
& \left(\int_{t}^{T} e^{4 \beta s}\|\widehat{y}(s)\|_{H}^{2}\|\widehat{z}(s)\|_{L_{2}(\Xi, H)}^{2} d s\right)^{1 / 2} \\
\leq & e^{2|\beta| T} \sup _{s \in[t, T]}\|\widehat{y}(s)\|_{H}\left(\int_{t}^{T}\|\widehat{z}(s)\|_{L_{2}(\Xi, H)}^{2} d s\right)^{1 / 2} \\
\leq & \frac{1}{2} e^{2|\beta| T} \sup _{s \in[t, T]}\|\widehat{y}(s)\|_{H}^{2}+\frac{1}{2} e^{2|\beta| T} \int_{t}^{T}\|\widehat{z}(s)\|_{L_{2}(\Xi, H)}^{2} d s .
\end{aligned}
$$

Since the assumption is that $y \in L_{\mathcal{P}}^{2}\left(\Omega ; L_{\beta}^{2}(H)\right)$, the right side of above inequality is integrable random variable and we can easily obtain $\mathbb{E} \sup _{s \in[t, T]} e^{2 \beta s}\|\widehat{y}(s)\|_{H}^{2}<\infty$. Then, stochastic integrals in 2.7) are integrable random variables. Taking expectation on both sides of 2.7 , we have

$$
\begin{aligned}
& e^{2 \beta t} \mathbb{E}\|\widehat{y}(t)\|_{H}^{2}-e^{2 \beta T} \mathbb{E}\|\widehat{y}(T)\|_{H}^{2}+2 \alpha \mathbb{E} \int_{t}^{T} e^{2 \beta s}\|\widehat{y}(s)\|_{V}^{2} d s \\
& +\left(2 \beta+2 \theta+2 \mu-2 \gamma-\frac{2 L^{2}}{\rho}+\frac{2 \mu}{e^{2 \beta \delta}}-\varepsilon\right) \mathbb{E} \int_{t}^{T} e^{2 \beta s}\|\widehat{y}(s)\|_{H}^{2} d s \\
& +\left(1-\rho-\frac{\rho}{e^{2 \beta \delta}}\right) \mathbb{E} \int_{t}^{T} e^{2 \beta s}\|\widehat{z}(s)\|_{L_{2}(\Xi, H)}^{2} d s \\
& \leq \frac{1}{\varepsilon} \mathbb{E} \int_{t}^{T} e^{2 \beta s}\|\widehat{f}(s)\|_{H}^{2} d s+\frac{\rho}{e^{2 \beta \delta}} \mathbb{E} \int_{T}^{T+\delta} e^{2 \beta s}\|\widehat{z}(s)\|_{L_{2}(\Xi, H)}^{2} d s \\
& -\frac{2 \mu}{e^{2 \beta \delta}} \mathbb{E} \int_{T}^{T+\delta} e^{2 \beta s}\|\widehat{y}(s)\|_{H}^{2} d s .
\end{aligned}
$$

We recall the assumption $\mathbb{E} \int_{0}^{\infty} e^{2 \beta s}\|y(s)\|_{H}^{2} d s<\infty, \mathbb{E} \int_{0}^{\infty} e^{2 \beta s}\|z(s)\|_{L_{2}(\Xi, H)}^{2} d s<\infty$, then we can find a sequence of $T_{n} \rightarrow \infty$, such that $e^{2 \beta T_{n}} \mathbb{E}\left\|\widehat{y}\left(T_{n}\right)\right\|_{H}^{2} \rightarrow 0, \int_{T_{n}}^{T_{n}+\delta} e^{2 \beta s}\|\widehat{y}(s)\|_{H}^{2} d s$ $\rightarrow 0$ and $\int_{T_{n}}^{T_{n}+\delta} e^{2 \beta s}\|\widehat{z}(s)\|_{L_{2}(\Xi, H)}^{2} d s \rightarrow 0$. Setting $T=T_{n}$ and letting $n \rightarrow \infty$, we obtain

$$
\begin{aligned}
& e^{2 \beta t} \mathbb{E}\|\widehat{y}(t)\|_{H}^{2}+2 \alpha \mathbb{E} \int_{t}^{\infty} e^{2 \beta s}\|\widehat{y}(s)\|_{V}^{2} d s \\
& +\left(2 \beta+2 \theta+2 \mu-2 \gamma-\frac{2 L^{2}}{\rho}+\frac{2 \mu}{e^{2 \beta \delta}}-\varepsilon\right) \mathbb{E} \int_{t}^{\infty} e^{2 \beta s}\|\widehat{y}(s)\|_{H}^{2} d s \\
& +\left(1-\rho-\frac{\rho}{e^{2 \beta \delta}}\right) \mathbb{E} \int_{t}^{\infty} e^{2 \beta s}\|\widehat{z}(s)\|_{L_{2}(\Xi, H)}^{2} d s \\
& \leq \frac{1}{\varepsilon} \mathbb{E} \int_{t}^{\infty} e^{2 \beta s}\|\widehat{f}(s)\|_{H}^{2} d s .
\end{aligned}
$$


In particular, we have

$$
\sup _{t \geq 0} e^{2 \beta t} \mathbb{E}\|\widehat{y}(t)\|_{H}^{2} \leq \frac{1}{\varepsilon} \mathbb{E} \int_{0}^{\infty} e^{2 \beta s}\|\widehat{f}(s)\|_{H}^{2} d s .
$$

Then, we obtain

$$
\begin{aligned}
& \sup _{t \geq 0} e^{2 \beta t} \mathbb{E}\|\widehat{y}(t)\|_{H}^{2}+2 \alpha \mathbb{E} \int_{0}^{\infty} e^{2 \beta s}\|\widehat{y}(s)\|_{V}^{2} d s \\
& +\left(1-\rho-\frac{\rho}{e^{2 \beta \delta}}\right) \mathbb{E} \int_{0}^{\infty} e^{2 \beta s}\|\widehat{z}(s)\|_{L_{2}(\Xi, H)}^{2} d s \\
& +\left(2 \beta+2 \theta+2 \mu-2 \gamma-\frac{2 L^{2}}{\rho}+\frac{2 \mu}{e^{2 \beta \delta}}-\varepsilon\right) \mathbb{E} \int_{0}^{\infty} e^{2 \beta s}\|\widehat{y}(s)\|_{H}^{2} d s \\
& \leq \frac{2}{\varepsilon} \mathbb{E} \int_{0}^{\infty} e^{2 \beta s}\|\widehat{f}(s)\|_{H}^{2} d s
\end{aligned}
$$

Letting $\rho<\frac{e^{2 \beta \delta}}{1+e^{2 \beta \delta}}$ close enough to $\frac{e^{2 \beta \delta}}{1+e^{2 \beta \delta}}$, for every $\beta+\mu-\gamma-\frac{L^{2}\left(1+e^{2 \beta \delta}\right)}{e^{2 \beta \delta}}+\frac{\mu}{e^{2 \beta \delta}}>-\bar{\theta}$, and $\theta>\bar{\theta}$, we can find $\varepsilon>0$ small enough such that $\beta+\mu-\gamma-\frac{L^{2}\left(1+e^{2 \beta \delta}\right)}{e^{2 \beta \delta}}+\frac{\mu}{e^{2 \beta \delta}}-\varepsilon>-\bar{\theta}$. Then there exists a constant $C_{0}>0$ such that

$$
\begin{aligned}
& \sup _{s \geq 0} e^{2 \beta s} \mathbb{E}\|\widehat{y}(s)\|_{H}^{2}+(\theta-\bar{\theta})\|\widehat{y}(s)\|_{L_{\mathcal{P}}^{2}\left(\Omega ; L_{\beta}^{2}(H)\right)} \\
& \quad+\|\widehat{z}(s)\|_{L_{\mathcal{P}}^{2}\left(\Omega ; L_{\beta}^{2}\left(L_{2}(\Xi, H)\right)\right)}+\|\widehat{y}(s)\|_{L_{\mathcal{P}}^{2}\left(\Omega ; L_{\beta}^{2}(V)\right)} \\
& \leq C_{0}\|\widehat{f}(s)\|_{L_{\mathcal{P}}^{2}\left(\Omega ; L_{\beta}^{2}(H)\right)},
\end{aligned}
$$

where $C_{0}$ depends only on $\beta, \mu, \gamma, L, \alpha$ and $\bar{\theta}$.

Next, taking expectation on both sides of (2.7), we can find a sequence of $T_{n} \rightarrow \infty$, such that $e^{2 \beta T_{n}} \mathbb{E}\left\|\widehat{y}\left(T_{n}\right)\right\|_{H}^{2} \rightarrow 0, \int_{T_{n}}^{T_{n}+\delta} e^{2 \beta s}\|\widehat{y}(s)\|_{H}^{2} d s \rightarrow 0$ and $\int_{T_{n}}^{T_{n}+\delta} e^{2 \beta s}\|\widehat{z}(s)\|_{L_{2}(\Xi, H)}^{2} d s \rightarrow 0$. Setting $T=T_{n}$ and letting $n \rightarrow \infty$, as shown previously, we have

$$
\begin{aligned}
& e^{2 \beta t} \mathbb{E}\|\widehat{y}(t)\|_{H}^{2}+2 \alpha \mathbb{E} \int_{t}^{\infty} e^{2 \beta s}\|\widehat{y}(s)\|_{V}^{2} d s \\
& +\left(2 \beta+2 \theta+2 \mu-2 \gamma-\frac{2 L^{2}}{\rho}+\frac{2 \mu}{e^{2 \beta \delta}}\right) \mathbb{E} \int_{t}^{\infty} e^{2 \beta s}\|\widehat{y}(s)\|_{H}^{2} d s \\
& +\left(1-\rho-\frac{\rho}{e^{2 \beta \delta}}\right) \mathbb{E} \int_{t}^{\infty} e^{2 \beta s}\|\widehat{z}(s)\|_{L_{2}(\Xi, H)}^{2} d s \\
& \leq 2 \mathbb{E} \int_{t}^{\infty} e^{2 \beta s}(\widehat{y}(s), \widehat{f}(s))_{H} d s,
\end{aligned}
$$

and by the Hölder inequality and (2.8), we obtain

$$
\begin{aligned}
\mathbb{E} \int_{t}^{\infty} e^{2 \beta s}(\widehat{y}(s), \widehat{f}(s))_{H} d s & \leq\left(\mathbb{E} \int_{t}^{\infty} e^{2 \beta s}\|\widehat{y}(s)\|_{H}^{2} d s\right)^{1 / 2}\left(\mathbb{E} \int_{t}^{\infty} e^{2 \beta s}\|\widehat{f}(s)\|_{H}^{2} d s\right)^{1 / 2} \\
& \leq \frac{C_{0}^{1 / 2}}{(\theta-\bar{\theta})^{1 / 2}} \mathbb{E} \int_{t}^{\infty} e^{2 \beta s}\|\widehat{f}(s)\|_{H}^{2} d s
\end{aligned}
$$


then we have

$$
\begin{aligned}
& (\theta-\bar{\theta})^{1 / 2} \sup _{s \geq 0} e^{2 \beta s} \mathbb{E}\|\widehat{y}(s)\|_{H}^{2}+(\theta-\bar{\theta})\|\widehat{y}(s)\|_{L_{\mathcal{P}}^{2}\left(\Omega ; L_{\beta}^{2}(H)\right)} \\
& +(\theta-\bar{\theta})^{1 / 2}\|\widehat{z}(s)\|_{L_{\mathcal{P}}^{2}\left(\Omega ; L_{\beta}^{2}\left(L_{2}(\Xi, H)\right)\right)}+(\theta-\bar{\theta})^{1 / 2}\|\widehat{y}(s)\|_{L_{\mathcal{P}}^{2}\left(\Omega ; L_{\beta}^{2}(V)\right)} \\
& \leq C_{1}\|\widehat{f}(s)\|_{L_{\mathcal{P}}^{2}\left(\Omega ; L_{\beta}^{2}(H)\right)},
\end{aligned}
$$

where $C_{1}$ depends only on $\beta, \mu, \gamma, L, \alpha$ and $\bar{\theta}$.

In the linear case $\phi=0$, we easily obtain

Corollary 2.11. Suppose that Assumption 2.2(ii), (iii) and Assumption 2.8 hold for some $\beta \in \mathbb{R}, \theta \in \mathbb{R}$. If $(y(\cdot), z(\cdot))$ is the solution to the following 2.9 corresponding to the generator $\left(A^{*}, f\right)$ :

$$
\begin{aligned}
y(T)-y(t)= & \int_{t}^{T} A^{*}(s) y(s) d s+\int_{t}^{T} \mathbb{E}\left[\theta y(s)-f(s) \mid \mathcal{F}_{s}\right] d s \\
& +\int_{t}^{T} z(s) d W(s), \quad 0 \leq t \leq T<\infty .
\end{aligned}
$$

Suppose that $(\bar{y}(\cdot), \bar{z}(\cdot))$ is the solution to the 2.9 corresponding to another generator $\left(A^{*}, \bar{f}\right)$, then for every $\bar{\theta}>-\beta+\gamma$ and $\theta>\bar{\theta}$, we have

$$
\begin{aligned}
& (\theta-\bar{\theta})^{1 / 2}\|z(s)-\bar{z}(s)\|_{L_{\mathcal{P}}^{2}\left(\Omega ; L_{\beta}^{2}\left(L_{2}(\Xi, H)\right)\right)}+(\theta-\bar{\theta})\|y(s)-\bar{y}(s)\|_{L_{\mathcal{P}}^{2}\left(\Omega ; L_{\beta}^{2}(H)\right)} \\
& +(\theta-\bar{\theta})^{1 / 2} \sup _{s \geq 0} e^{2 \beta s} \mathbb{E}\|y(s)-\bar{y}(s)\|_{H}^{2}+(\theta-\bar{\theta})^{1 / 2}\|y(s)-\bar{y}(s)\|_{L_{\mathcal{P}}^{2}\left(\Omega ; L_{\beta}^{2}(V)\right)} \\
\leq & C_{2}\|f(s)-\bar{f}(s)\|_{L_{\mathcal{P}}^{2}\left(\Omega ; L_{\beta}^{2}(H)\right)},
\end{aligned}
$$

where $C_{2}$ depends only on $\beta, \gamma, \alpha$ and $\bar{\theta}$.

The next step to Theorem 2.13 is to prove that for the existence of large value $\theta$. Let's introduce the following lemma.

Lemma 2.12. Suppose that Assumption 2.2(ii), (iii) and Assumption 2.8 hold for $f(\cdot) \in$ $L_{\mathcal{P}}^{2}\left(\Omega ; L_{\beta}^{2}(H)\right)$, some $\beta \in \mathbb{R}$ and $\theta \in \mathbb{R}$. Then for every $\bar{\theta}>-\beta+\gamma$ and $\theta>\bar{\theta}$, the $B S E E$

$$
\begin{aligned}
y(T)-y(t)= & \int_{t}^{T}(\theta y(s)-f(s)) d s+\int_{t}^{T} A^{*}(s) y(s) d s \\
& +\int_{t}^{T} z(s) d W(s), \quad 0 \leq t \leq T<\infty
\end{aligned}
$$

admits a unique solution $(y(\cdot), z(\cdot)) \in L_{\mathcal{P}}^{2}\left(\Omega ; L_{\beta}^{2}(H)\right) \times L_{\mathcal{P}}^{2}\left(\Omega ; L_{\beta}^{2}\left(L_{2}(\Xi, H)\right)\right)$. 
Proof. By Corollary 2.11, the uniqueness of 2.10 can be easily obtained. To show the existence, we establish a solution as follows. In detail, for $n=1,2, \ldots$, we define

$$
f_{n}(s)=\mathbb{1}_{[0, n]}(s) f(s), \quad s \in[0, \infty) .
$$

It is obvious that the sequence $\left\{f_{n}(s)\right\}_{n=1}^{\infty}$ converges to $\{f(s)\}$ in $L_{\mathcal{P}}^{2}\left(\Omega ; L_{\beta}^{2}(H)\right)$. By Theorem 2.4 in [17], for each $n$, let $\left(\bar{y}_{n}(s), \bar{z}_{n}(s)\right)$ be the unique solution of the following finite horizon BSEE:

$$
\bar{y}(n)-\bar{y}(t)=\int_{t}^{n}(\theta \bar{y}(s)-f(s)) d s+\int_{t}^{n} A^{*}(s) \bar{y}(s) d s+\int_{t}^{n} \bar{z}(s) d W(s), \quad t \in[0, n] .
$$

Moreover, we define

$$
\left(y_{n}(s), z_{n}(s)\right):= \begin{cases}\left(\bar{y}_{n}(s), \bar{z}_{n}(s)\right), & s \in[0, n] \\ (0,0), & s \in(n, \infty) .\end{cases}
$$

We easily get $\left(y_{n}(s), z_{n}(s)\right) \in L_{\mathcal{P}}^{2}\left(\Omega ; L_{\beta}^{2}(V)\right) \times L_{\mathcal{P}}^{2}\left(\Omega ; L_{\beta}^{2}\left(L_{2}(\Xi, H)\right)\right)$, then $\left(y_{n}(s), z_{n}(s)\right)$ is the solution of the following BSEE:

$$
\begin{aligned}
y_{n}(T)-y_{n}(t)= & \int_{t}^{T}\left(\theta y_{n}(s)-f_{n}(s)\right) d s+\int_{t}^{T} A^{*}(s) y_{n}(s) d s \\
& +\int_{t}^{T} z_{n}(s) d W(s), \quad 0 \leq t \leq T<\infty .
\end{aligned}
$$

By Corollary 2.11, we obtain

$$
\begin{aligned}
& (\theta-\bar{\theta})^{1 / 2}\left\|z_{n}(s)-z_{m}(s)\right\|_{L_{\mathcal{P}}^{2}\left(\Omega ; L_{\beta}^{2}\left(L_{2}(\Xi, H)\right)\right)}+(\theta-\bar{\theta})\left\|y_{n}(s)-y_{m}(s)\right\|_{L_{\mathcal{P}}^{2}\left(\Omega ; L_{\beta}^{2}(H)\right)} \\
& +(\theta-\bar{\theta})^{1 / 2} \sup _{s \geq 0} e^{2 \beta s} \mathbb{E}\left\|y_{n}(s)-y_{m}(s)\right\|_{H}^{2}+(\theta-\bar{\theta})^{1 / 2}\left\|y_{n}(s)-y_{m}(s)\right\|_{L_{\mathcal{P}}^{2}\left(\Omega ; L_{\beta}^{2}(V)\right)} \\
\leq & C_{2}\left\|f_{n}(s)-f_{m}(s)\right\|_{L_{\mathcal{P}}^{2}\left(\Omega ; L_{\beta}^{2}(H)\right)},
\end{aligned}
$$

which means $\left\{\left(y_{n}(s), z_{n}(s)\right)\right\}_{n=1}^{\infty}$ is a Cauchy sequence in above space. We denote by $(y(s), z(s))$ the limit of $\left\{\left(y_{n}(s), z_{n}(s)\right)\right\}_{n=1}^{\infty}$ in $L_{\mathcal{P}}^{2}\left(\Omega ; L_{\beta}^{2}(V)\right) \times L_{\mathcal{P}}^{2}\left(\Omega ; L_{\beta}^{2}\left(L_{2}(\Xi, H)\right)\right)$, and shall show that $(y(s), z(s))$ satisfies the BSEE $P$-a.s.,

$$
\begin{aligned}
y(T)-y(t)= & \int_{t}^{T}(\theta y(s)-f(s)) d s+\int_{t}^{T} A^{*}(s) y(s) d s \\
& +\int_{t}^{T} z(s) d W(s), \quad 0 \leq t \leq T<\infty .
\end{aligned}
$$

We deduce that

$$
\begin{aligned}
\mathbb{E}\left\|\int_{t}^{T}\left(z_{n}(s)-z(s)\right) d W(s)\right\|_{L_{2}(\Xi, H)}^{2} & =\mathbb{E} \int_{t}^{T}\left\|z_{n}(s)-z(s)\right\|_{L_{2}(\Xi, H)}^{2} d s \\
& \leq e^{2|\beta| T} \mathbb{E} \int_{0}^{\infty} e^{2 \beta s}\left\|z_{n}(s)-z(s)\right\|_{L_{2}(\Xi, H)}^{2} d s \\
& \rightarrow 0 \quad \text { as } n \rightarrow \infty,
\end{aligned}
$$


i.e., the item $\int_{t}^{T} z_{n}(s) d W(s)$ converges to $\int_{t}^{T} z(s) d W(s)$ in $L^{2}\left(\Omega, \mathcal{F}, P ; L_{2}(\Xi, H)\right)$, which is the space of $\mathcal{F}$-measurable square integrable random variables. Then, the same argument also leads to a similar conclusion: the item $\int_{t}^{T}\left(\theta y_{n}(s)-f_{n}(s)\right) d s$ converges to $\int_{t}^{T}(\theta y(s)-$ $f(s)) d s$ in $L^{2}(\Omega, \mathcal{F}, P ; H)$. By Assumption 2.2(iii), we obtain

$\mathbb{E}\left\|\int_{t}^{T} A^{*}\left(y_{n}(s)-y(s)\right) d s\right\|_{V^{*}}^{2} \leq e^{2|\beta| T} \mathbb{E} \int_{0}^{\infty} \bar{C}^{2} e^{2 \beta s}\left\|y_{n}(s)-y(s)\right\|_{V}^{2} d s \rightarrow 0 \quad$ as $n \rightarrow \infty$,

i.e., the item $\int_{t}^{T} A^{*} y_{n}(s) d s$ converges to $\int_{t}^{T} A^{*} y(s) d s$ in $L^{2}\left(\Omega, \mathcal{F}, P ; V^{*}\right)$.

After that, since $\lim _{n \rightarrow \infty} \mathbb{E}\left[\int_{0}^{\infty} e^{2 \beta s}\left\|y_{n}(s)-y(s)\right\|_{H}^{2} d s\right]=0$, there exists a subsequence of $\left\{y_{n}(s)\right\}_{n=1}^{\infty}$ still converges to $\{y(s)\}$ for a.e. $s \in[0, \infty)$. On the other hand, by $\lim _{n, m \rightarrow \infty} \sup _{s \geq 0} e^{2 \beta s} \mathbb{E}\left\|y_{n}(s)-y_{m}(s)\right\|_{H}^{2}=0$, for every $s \in[0, \infty)$, there exists a $\bar{y}(s) \in L^{2}(\Omega, \mathcal{F}, P ; H)$ such that $y_{n}(s) \rightarrow \bar{y}(s)$ in $L^{2}(\Omega, \mathcal{F}, P ; H)$ as $n \rightarrow \infty$. Then we have $y(s)=\bar{y}(s)$ for almost all $s \in[0, \infty)$. We identify $y(s)$ with $\bar{y}(s)$. Thus, the proof is completed.

Theorem 2.13. Suppose that Assumption 2.2(ii), (iii) and Assumption 2.8 hold for $f(\cdot) \in$ $L_{\mathcal{P}}^{2}\left(\Omega ; L_{\beta}^{2}(H)\right)$, some $\beta \in \mathbb{R}$ and $\theta \in \mathbb{R}$. Then for $\bar{\theta}>-\left(\beta+\mu-\gamma-\frac{L^{2}\left(1+e^{2 \beta \delta}\right)}{e^{2 \beta \delta}}+\frac{\mu}{e^{2 \beta \delta}}\right)$ and $\theta>\bar{\theta}$, the equation (2.5) admits a unique solution $(y(\cdot), z(\cdot))$ such that

$$
y \in L_{\mathcal{P}}^{2}\left(\Omega ; L_{\beta}^{2}(H)\right), \quad z \in L_{\mathcal{P}}^{2}\left(\Omega ; L_{\beta}^{2}\left(L_{2}(\Xi, H)\right)\right) .
$$

Proof. We prove the result by using the method in Theorem 3.7 of [14. For simplicity, we define the space

$$
\mathcal{T}_{\beta}^{2}:=L_{\mathcal{P}}^{2}\left(\Omega ; L_{\beta}^{2}(H)\right) \times L_{\mathcal{P}}^{2}\left(\Omega ; L_{\beta}^{2}\left(L_{2}(\Xi, H)\right)\right)
$$

endowed with the norm $\|(y, z)\|_{\mathcal{T}_{\beta}^{2}}=\|y\|_{L_{\mathcal{P}}^{2}\left(\Omega ; L_{\beta}^{2}(H)\right)}+\|z\|_{L_{\mathcal{P}}^{2}\left(\Omega ; L_{\beta}^{2}\left(L_{2}(\Xi, H)\right)\right)}$. We define a mapping $\Gamma: \mathcal{T}_{\beta}^{2} \rightarrow \mathcal{T}_{\beta}^{2}$, letting $(y(s), z(s))=\Gamma(u(s), v(s))$ if $(y(s), z(s))$ is the solution of this equation, $P$-a.s.,

$$
\begin{aligned}
y(T)-y(t)= & \int_{t}^{T} A^{*}(s) y(s) d s \\
& +\int_{t}^{T} \mathbb{E}\left[\phi(s, u(s), u(s+\delta), v(s), v(s+\delta))+\theta y(s)-f(s) \mid \mathcal{F}_{s}\right] d s \\
& +\int_{t}^{T} z(s) d W(s), \quad 0 \leq t \leq T<\infty .
\end{aligned}
$$

If there exist $(u(s), v(s)),(\bar{u}(s), \bar{v}(s)) \in \mathcal{T}_{\beta}^{2},(y(s), z(s))=\Gamma(u(s), v(s)),(\bar{y}(s), \bar{z}(s))=$ $\Gamma(\bar{u}(s), \bar{v}(s))$, by Corollary 2.11, we obtain

$$
(\theta-\bar{\theta})^{1 / 2}\|z(s)-\bar{z}(s)\|_{L_{\mathcal{P}}^{2}\left(\Omega ; L_{\beta}^{2}\left(L_{2}(\Xi, H)\right)\right)}+(\theta-\bar{\theta})\|y(s)-\bar{y}(s)\|_{L_{\mathcal{P}}^{2}\left(\Omega ; L_{\beta}^{2}(H)\right)}
$$




$$
\begin{aligned}
\leq C_{2} \mathbb{E}\left(\int_{0}^{\infty}\right. & e^{2 \beta s} \mathbb{E}[\| \phi(s, u(s), u(s+\delta), v(s), v(s+\delta)) \\
& \left.\left.-\phi(s, \bar{u}(s), \bar{u}(s+\delta), \bar{v}(s), \bar{v}(s+\delta)) \|_{H}^{2} \mid \mathcal{F}_{s}\right] d s\right)^{1 / 2}
\end{aligned}
$$

and then, by the Lipschitz condition on $\phi$, for some positive constant $c$ independent of $\theta$, we obtain

$$
\begin{aligned}
& (\theta-\bar{\theta})\|y(s)-\bar{y}(s)\|_{L_{\mathcal{P}}^{2}\left(\Omega ; L_{\beta}^{2}(H)\right)}+(\theta-\bar{\theta})^{1 / 2}\|z(s)-\bar{z}(s)\|_{L_{\mathcal{P}}^{2}\left(\Omega ; L_{\beta}^{2}\left(L_{2}(\Xi, H)\right)\right)} \\
\leq & c\|u(s)-\bar{u}(s)\|_{L_{\mathcal{P}}^{2}\left(\Omega ; L_{\beta}^{2}(H)\right)}+c\|v(s)-\bar{v}(s)\|_{L_{\mathcal{P}}^{2}\left(\Omega ; L_{\beta}^{2}\left(L_{2}(\Xi, H)\right)\right)} .
\end{aligned}
$$

Therefore, $\Gamma$ is a contraction of $\mathcal{T}$, for all $\theta$ that is large enough. Its unique fixed point is the solution.

Next, we fix $\bar{\theta}>-\left(\beta+\mu-\gamma-\frac{L^{2}\left(1+e^{2 \beta \delta}\right)}{e^{2 \beta \delta}}+\frac{\mu}{e^{2 \beta \delta}}\right)$ and define $K$ as the set of those real numbers $\theta>\bar{\theta}$ such that for every $f \in L_{\mathcal{P}}^{2}\left(\Omega ; L_{\beta}^{2}(H)\right)$ there exists a unique solution $(y(s), z(s)) \in \mathcal{T}_{\beta}^{2}$ corresponding to $\theta$ and $f$. Letting $C_{1}$ be the constant whose existence is asserted in Theorem 2.10, we also set

$$
C_{1}(\theta)=C_{1}\left\{(\theta-\bar{\theta})^{-1} \vee(\theta-\bar{\theta})^{-1 / 2}\right\}
$$

We argue that if $K$ contains a number $\theta_{0}$, then it contains every number $\theta>\bar{\theta}$ belonging to the $\left(\theta_{0}-C_{1}\left(\theta_{0}\right)^{-1}, \theta_{0}+C_{1}\left(\theta_{0}\right)^{-1}\right)$. In fact, for any $\theta>\bar{\theta}$, we can define a mapping $\Gamma: \mathcal{T}_{\beta}^{2} \rightarrow \mathcal{T}_{\beta}^{2}$, and setting $(y(s), z(s))=\Gamma(u(s), v(s))$ if $(y(s), z(s))$ is the solution of the equation, $P$-a.s.,

$$
\begin{aligned}
y(T)-y(t)= & \int_{t}^{T} A^{*}(s) y(s) d s+\int_{t}^{T} \mathbb{E}\left[\phi(s, y(s), y(s+\delta), z(s), z(s+\delta)) \mid \mathcal{F}_{s}\right] d s \\
& +\int_{t}^{T} z(s) d W(s)+\theta_{0} \int_{t}^{T} \mathbb{E}\left[y(s) \mid \mathcal{F}_{s}\right] d s \\
& -\int_{t}^{T}\left[\left(\theta_{0}-\theta\right) u(s)+f(s)\right] d s, \quad 0 \leq t \leq T<\infty .
\end{aligned}
$$

Therefore, an element $(y(s), z(s)) \in \mathcal{T}_{\beta}^{2}$ is a solution of 2.5 if and only if $(y(s), z(s))$ is a fixed point of $\Gamma$; if, for $(u(s), v(s)),(\bar{u}(s), \bar{v}(s)) \in \mathcal{T}_{\beta}^{2}$ and $(y(s), z(s))=\Gamma(u(s), v(s))$, $(\bar{y}(s), \bar{z}(s))=\Gamma(\bar{u}(s), \bar{v}(s))$, then, by Theorem 2.10, we obtain

$$
\begin{aligned}
\|(y(s)-\bar{y}(s), z(s)-\bar{z}(s))\|_{\mathcal{T}_{\beta}^{2}} & \leq C_{1}\left(\theta_{0}\right)\left|\theta-\theta_{0}\right| \cdot\|u(s)-\bar{u}(s)\|_{L_{\mathcal{P}}^{2}\left(\Omega ; L_{\beta}^{2}(H)\right)} \\
& \leq C_{1}\left(\theta_{0}\right)\left|\theta-\theta_{0}\right| \cdot\|(u(s)-\bar{u}(s), v(s)-\bar{v}(s))\|_{\mathcal{T}_{\beta}^{2}},
\end{aligned}
$$

this shows that $\Gamma$ is a contraction if $C_{1}\left(\theta_{0}\right)\left|\theta-\theta_{0}\right|<1$, and the claim follows immediately from the Banach contraction principle. 


\section{Stochastic maximum principle}

\subsection{Optimal control problem on infinite horizon}

In this subsection, we study a class of infinite horizon optimal control problems. Now, we introduce the controlled SDEE in the Gelfand triple $\left(V, H, V^{*}\right)$ as follows:

$$
\begin{aligned}
x(t)= & \varphi(0)-\int_{0}^{t} A(s) x(s) d s+\int_{0}^{t} h(s, x(s), x(s-\delta), u(s), u(s-\delta)) d s \\
& +\int_{0}^{t} g(s, x(s), x(s-\delta), u(s), u(s-\delta)) d W(s), \quad t \in[0, \infty), \\
x(t)= & \varphi(t), \quad t \in[-\delta, 0], \\
u(t)= & u_{0}(t), \quad t \in[-\delta, 0],
\end{aligned}
$$

with the cost functional

$$
J(u(\cdot))=\mathbb{E}\left[\int_{0}^{\infty} e^{-\lambda s} k(s, x(s), x(s-\delta), u(s), u(s-\delta)) d s\right],
$$

where the operator $A: \Omega \times[0, \infty) \rightarrow L\left(V, V^{*}\right)$ is $\mathcal{P} / \mathcal{B}\left(L\left(V, V^{*}\right)\right)$-measurable; the mapping $h:[0, \infty) \times \Omega \times H \times H \times \mathcal{U} \times \mathcal{U} \rightarrow H$ and $g:[0, \infty) \times \Omega \times H \times H \times \mathcal{U} \times \mathcal{U} \rightarrow$ $L_{2}(\Xi, H)$ are $\mathcal{P} \otimes \mathcal{B}(H) \otimes \mathcal{B}(H) \otimes \mathcal{B}(\mathcal{U}) \otimes \mathcal{B}(\mathcal{U}) / \mathcal{B}(H)$-measurable and $\mathcal{P} \otimes \mathcal{B}(H) \otimes \mathcal{B}(H) \otimes$ $\mathcal{B}(\mathcal{U}) \otimes \mathcal{B}(\mathcal{U}) / \mathcal{B}\left(L_{2}(\Xi, H)\right)$-measurable, respectively; the mapping $\varphi:[-\delta, 0] \times \Omega \rightarrow H$ is $\mathcal{P}_{[-\delta, 0]} / \mathcal{B}(H)$-measurable; the mapping $u_{0}:[-\delta, 0] \times \Omega \rightarrow \mathcal{U}$ is $\mathcal{P}_{[-\delta, 0]} / \mathcal{B}(\mathcal{U})$-measurable; $\lambda$ is a given attenuation coefficient and large enough; the mapping $k:[0, \infty) \times \Omega \times H \times$ $H \times \mathcal{U} \times \mathcal{U} \rightarrow \mathbb{R}$ is $\mathcal{P} \otimes \mathcal{B}(H) \otimes \mathcal{B}(H) \otimes \mathcal{B}(\mathcal{U}) \otimes \mathcal{B}(\mathcal{U}) / \mathcal{B}(\mathbb{R})$-measurable. Here, $\mathcal{U}$ denotes the control domain and is a nonempty convex closed subset of a real separable Hilbert space $U$. In addition, we assume that the control process $u(\cdot) \triangleq\{u(s), 0 \leq s<\infty\}$ is $\mathbb{F}$-predictable. For brevity, we write $x(s+\delta)=x(s-\delta), u(s+\delta)=u(s-\delta)$.

We define the set of all admissible control processes as follows, and $\beta$ will be given later,

$$
U_{\mathrm{ad}}=\left\{u(\cdot) \in L_{\mathcal{P}}^{2}\left(\Omega ; L_{\beta}^{2}(U)\right): u(s) \in \mathcal{U}\right\},
$$

where $u(\cdot)$ is called the control process, if $u(\cdot) \in U_{\text {ad }}$.

Moreover, we introduce the following assumptions:

Assumption 3.1. (i) $u_{0}(\cdot) \in L_{\mathcal{P}}^{2}\left([0,-\delta] \times \Omega ; L_{\beta}^{2}(U)\right)$ and $u_{0}(s) \in \mathcal{U}, \varphi(\cdot) \in L_{\mathcal{P}}^{2}(\Omega$; $C[-\delta, 0] ; H), h(\cdot, 0,0,0,0) \in L_{\mathcal{P}}^{2}\left(\Omega ; L_{\beta}^{2}(H)\right)$ and $g(\cdot, 0,0,0,0) \in L_{\mathcal{P}}^{2}\left(\Omega ; L_{\beta}^{2}\left(L_{2}(\Xi, H)\right)\right) ;$

(ii) The operator A satisfies the coercivity and boundedness conditions, i.e., (ii) and (iii) in Assumption 2.2. 
(iii) For almost all $(s, \omega) \in[0, \infty) \times \Omega, h$ and $g$ are Gâteaux differentiable with respect to $\left(x, x_{\delta}, u, u_{\delta}\right)$ with continuous and uniformly bounded Gâteaux derivatives $h_{x}, h_{x_{\delta}}$, $g_{x}, g_{x_{\delta}}, h_{u}, h_{u_{\delta}}, g_{u}, g_{u_{\delta}}$, i.e., there exists a constant $M>0$ such that

$$
\sup _{\left(s, \omega, x, x_{\delta}, u, u_{\delta}\right) \in[0, \infty) \times \Omega \times H \times H \times \mathcal{U} \times \mathcal{U}}\left\|h_{x}\left(s, x, x_{\delta}, u, u_{\delta}\right)\right\|_{L(H)} \leq M,
$$

and $h_{x_{\delta}}, g_{x}, g_{x_{\delta}}, h_{u}, h_{u_{\delta}}, g_{u}, g_{u_{\delta}}$ are similar to the above conditions;

(iv) For almost all $(s, \omega) \in[0, \infty) \times \Omega, k$ is Gâteaux differentiable with respect to $\left(x, x_{\delta}, u\right.$, $\left.u_{\delta}\right)$ with continuous Gâteaux derivatives $k_{x}, k_{x_{\delta}}, k_{u}, k_{u_{\delta}}$. Furthermore, for almost all $(s, \omega) \in[0, \infty) \times \Omega$, there exists a constant $C>0$ such that for all $\left(x, x_{\delta}, u, u_{\delta}\right) \in$ $H \times H \times \mathcal{U} \times \mathcal{U}$,

$$
\begin{gathered}
\left|k\left(s, x, x_{\delta}, u, u_{\delta}\right)\right| \leq C\left(1+\|x\|_{H}^{2}+\left\|x_{\delta}\right\|_{H}^{2}+\|u\|_{U}^{2}+\left\|u_{\delta}\right\|_{U}^{2}\right), \\
\left\|k_{x}\left(s, x, x_{\delta}, u, u_{\delta}\right)\right\|_{H}+\left\|k_{x_{\delta}}\left(s, x, x_{\delta}, u, u_{\delta}\right)\right\|_{H} \\
\quad+\left\|k_{u}\left(s, x, x_{\delta}, u, u_{\delta}\right)\right\|_{U}+\left\|k_{u_{\delta}}\left(s, x, x_{\delta}, u, u_{\delta}\right)\right\|_{U} \\
\leq C\left(1+\|x\|_{H}+\left\|x_{\delta}\right\|_{H}+\|u\|_{U}+\left\|u_{\delta}\right\|_{U}\right) .
\end{gathered}
$$

By Theorem 2.5, it is clear that by Assumption 3.1 the SDEE (3.1) admits a unique solution $x(\cdot) \in L_{\mathcal{P}}^{2}\left(\Omega ; L_{\beta}^{2}(H)\right) \cap L_{\mathcal{P}}^{2}\left(\Omega ; C_{\beta}(H)\right)$ for $\beta<-\left(\gamma+19 M^{2}+1 / 2\right) \wedge 0$ and $u(\cdot) \in U_{\text {ad }}$. We denote $x(\cdot) \triangleq x^{u}(\cdot)$ the state process corresponding to the control process $u(\cdot)$ and call $(u(\cdot) ; x(\cdot))$ the admissible pair. Moreover, Assumption 3.1(iv) and the equation (2.3) imply that $|J(u(\cdot))|<\infty$, for $\lambda \geq-2 \beta$.

An optimal control problem can be stated as follows:

Problem 3.2. Find an admissible control process $\bar{u}(\cdot) \in U_{\text {ad }}$ such that

$$
J(\bar{u}(\cdot))=\inf _{u(\cdot) \in U_{\mathrm{ad}}} J(u(\cdot)) .
$$

Any admissible control $\bar{u}(\cdot)$ satisfying (3.3) is called an optimal control process. Thus, the state process $\bar{x}(\cdot)$ associated with $\bar{u}(\cdot)$ is called an optimal state process, and then $(\bar{u}(\cdot) ; \bar{x}(\cdot))$ is called an optimal pair.

We define the Hamiltonian $\mathcal{H}:[0, \infty) \times \Omega \times H \times H \times \mathcal{U} \times \mathcal{U} \times H \times L_{2}(\Xi, H) \rightarrow \mathbb{R}$ of Problem 3.2 as follows:

$$
\begin{aligned}
\mathcal{H}\left(s, x, x_{\delta}, u, u_{\delta}, p, q\right):= & \left(h\left(s, x, x_{\delta}, u, u_{\delta}\right)-\lambda x, p\right)_{H} \\
& +\left(g\left(s, x, x_{\delta}, u, u_{\delta}\right), q\right)_{L_{2}(\Xi, H)}+k\left(s, x, x_{\delta}, u, u_{\delta}\right) .
\end{aligned}
$$

From Assumption 3.1(iii) and (iv), it is obvious that the Hamiltonian $\mathcal{H}$ is also continuously differentiable with respect to $\left(x, x_{\delta}, u, u_{\delta}\right)$. Write $\mathcal{H}_{x}, \mathcal{H}_{x_{\delta}}, \mathcal{H}_{u}$ and $\mathcal{H}_{u_{\delta}}$ for the corresponding Gâteaux derivatives. 
For any admissible pair $(u(\cdot) ; x(\cdot))$, we introduce the following adjoint equation of the controlled system (3.1) and (3.2) governing the unknown $\mathbb{F}$-adapted processes $\{p(s), s \in$ $[0, \infty)\}$ and $\{q(s), s \in[0, \infty)\}$

$$
d p(s)=\left\{A^{*}(s) p(s)-\mathcal{H}_{x}(s)-e^{-\lambda \delta} \mathbb{E}\left[\mathcal{H}_{x_{\delta}}(s+\delta) \mid \mathcal{F}_{s}\right]\right\} d s+q(s) d W(s), \quad s \in[0, \infty),
$$

where we denote by

$$
\mathcal{H}(s) \triangleq \mathcal{H}(s, x(s), x(s-\delta), u(s), u(s-\delta), p(s), q(s))
$$

where $A^{*}$ is the adjoint operator of $A$. In fact, the adjoint equation (3.5) is a linear ABSEE. By Theorem 2.13, it is clear that the ABSEE (3.5) admits a unique solution $(p(\cdot), q(\cdot)) \in$ $L_{\mathcal{P}}^{2}\left(\Omega ; L_{\beta}^{2}(H)\right) \times L_{\mathcal{P}}^{2}\left(\Omega ; L_{\beta}^{2}\left(L_{2}(\Xi, H)\right)\right)$ for $\lambda>\left(-\beta+\gamma+\left(3 M / 2+M^{2}\right) \frac{1+e^{2 \beta \delta}}{e^{2 \beta \delta}}\right) \vee 0$. Especially, we call $(\bar{p}(\cdot), \bar{q}(\cdot))$ the adjoint process corresponding to the optimal control $\bar{u}(\cdot)$.

\subsection{Sufficient maximum principle}

In this subsection, we prove the sufficient maximum principle. For any control process $u(\cdot) \in U_{\text {ad }}$, let $(x(\cdot), p(\cdot), q(\cdot))$ be the corresponding solutions to the state equation (3.1) and the adjoint equation (3.5), respectively. In order to simplify the notation, we denote by

$$
\begin{aligned}
& G(s) \triangleq G(s, x(s), x(s-\delta), u(s), u(s-\delta)), \quad G:=h, g, \\
& \bar{G}(s) \triangleq G(s, \bar{x}(s), \bar{x}(s-\delta), \bar{u}(s), \bar{u}(s-\delta)), \quad G:=h, g, \\
& \mathcal{H}(s) \triangleq \mathcal{H}(s, x(s), x(s-\delta), u(s), u(s-\delta), \bar{p}(s), \bar{q}(s)), \\
& \overline{\mathcal{H}}(s) \triangleq \mathcal{H}(s, \bar{x}(s), \bar{x}(s-\delta), \bar{u}(s), \bar{u}(s-\delta), \bar{p}(s), \bar{q}(s)) .
\end{aligned}
$$

Moreover, we introduce the following lemma.

Lemma 3.3. Suppose that Assumption 3.1 holds for $\beta<-\left(\gamma+19 M^{2}+1 / 2\right) \wedge 0, \lambda>$ $\left(-\beta+\gamma+\left(3 M / 2+M^{2}\right) \frac{1+e^{2 \beta \delta}}{e^{2 \beta \delta}}\right) \vee(-2 \beta)$ then we have

$$
\begin{aligned}
& J(u(\cdot))-J(\bar{u}(\cdot)) \\
= & \mathbb{E}\left[\int_{0}^{\infty} e^{-\lambda s}\left\{\mathcal{H}(s)-\overline{\mathcal{H}}(s)-\left(\overline{\mathcal{H}}_{x}(s)+e^{-\lambda \delta} \overline{\mathcal{H}}_{x_{\delta}}(s+\delta), x(s)-\bar{x}(t)\right)_{H}\right\} d s\right] .
\end{aligned}
$$

Proof. From the cost functional $(3.2)$ and the Hamiltonian $\mathcal{H}(3.4)$, we have

$$
\begin{aligned}
& J(u(\cdot))-J(\bar{u}(\cdot)) \\
= & \mathbb{E}\left[\int_{0}^{\infty} e^{-\lambda s}\{k(s, x(s), x(s-\delta), u(s), u(s-\delta))-k(s, \bar{x}(s), \bar{x}(s-\delta), \bar{u}(s), \bar{u}(s-\delta))\} d s\right]
\end{aligned}
$$




$$
\begin{aligned}
& =\mathbb{E}\left[\int_{0}^{\infty} e^{-\lambda s}\left\{\mathcal{H}(s)-\overline{\mathcal{H}}(s)-(\bar{p}(s), h(s)-\bar{h}(s))_{H}-(\bar{q}(s), g(s)-\bar{g}(s))_{L_{2}(\Xi, H)}\right\} d s\right] \\
& \quad+\mathbb{E}\left[\int_{0}^{\infty} \lambda e^{-\lambda s}(\bar{p}(s), x(s)-\bar{x}(s))_{H} d s\right] .
\end{aligned}
$$

Applying Itô's formula to $\left(e^{-\lambda s} \bar{p}(s), x(s)-\bar{x}(s)\right)_{H}$ on the interval $[0, T]$, we obtain

$$
\begin{aligned}
& \left(e^{-\lambda T} \bar{p}(T), x(T)-\bar{x}(T)\right)_{H} \\
= & \int_{0}^{T} e^{-\lambda s}(\bar{p}(s), h(s)-\bar{h}(s))_{H} d s+\int_{0}^{T} e^{-\lambda s}(\bar{p}(s), g(s)-\bar{g}(s))_{H} d W(s) \\
& -\int_{0}^{T} \lambda e^{-\lambda s}(\bar{p}(s), x(s)-\bar{x}(s))_{H} d s+\int_{0}^{T} e^{-\lambda s}(\bar{q}(s), x(s)-\bar{x}(s))_{H} d W(s) \\
& +\int_{0}^{T} e^{-\lambda s}\left(x(s)-\bar{x}(s),-\mathcal{H}_{x}(s)-e^{-\lambda \delta} \mathbb{E}\left[\mathcal{H}_{x_{\delta}}(s+\delta) \mid \mathcal{F}_{s}\right]\right)_{H} d s \\
& +\int_{0}^{T} e^{-\lambda s}(\bar{q}(s), g(s)-\bar{g}(s))_{L_{2}(\Xi, H)} d s .
\end{aligned}
$$

Taking expectation on both sides of (3.7), we obtain

$$
\begin{aligned}
& \mathbb{E}\left[\left(e^{-\lambda T} \bar{p}(T), x(T)-\bar{x}(T)\right)_{H}\right]+\mathbb{E}\left[\int_{0}^{T} \lambda e^{-\lambda s}(\bar{p}(s), x(s)-\bar{x}(s))_{H} d s\right] \\
= & \mathbb{E}\left[\int_{0}^{T} e^{-\lambda s}(\bar{p}(s), h(s)-\bar{h}(s))_{H} d s\right]+\mathbb{E}\left[\int_{0}^{T} e^{-\lambda s}(\bar{q}(s), g(s)-\bar{g}(s))_{L_{2}(\Xi, H)} d s\right] \\
& +\mathbb{E}\left[\int_{0}^{T} e^{-\lambda s}\left(x(s)-\bar{x}(s),-\mathcal{H}_{x}(s)-e^{-\lambda \delta} \mathbb{E}\left[\mathcal{H}_{x_{\delta}}(s+\delta) \mid \mathcal{F}_{s}\right]\right)_{H} d s\right] .
\end{aligned}
$$

Noting that

$$
x(T), \bar{x}(T) \in L_{\mathcal{P}}^{2}\left(\Omega ; L_{\beta}^{2}(H)\right) \cap L_{\mathcal{P}}^{2}\left(\Omega ; C_{\beta}(H)\right), \quad \bar{p}(T) \in L_{\mathcal{P}}^{2}\left(\Omega ; L_{\beta}^{2}(H)\right),
$$

we can find a sequence of $T_{n} \rightarrow \infty$, such that $e^{-\lambda T_{n}} \mathbb{E}\left\|x\left(T_{n}\right)\right\|_{H}^{2} \rightarrow 0, e^{-\lambda T_{n}} \mathbb{E}\left\|\bar{x}\left(T_{n}\right)\right\|_{H}^{2} \rightarrow 0$ and $e^{-\lambda T_{n}} \mathbb{E}\left\|\bar{p}\left(T_{n}\right)\right\|_{H}^{2} \rightarrow 0$, as $n \rightarrow \infty$, therefore, we obtain

$$
\begin{aligned}
& \lim _{T_{n} \rightarrow \infty} \mathbb{E}\left[\left(e^{-\lambda T_{n}} \bar{p}\left(T_{n}\right), x\left(T_{n}\right)-\bar{x}\left(T_{n}\right)\right)_{H}\right] \\
\leq & \lim _{T_{n} \rightarrow \infty} \mathbb{E}\left[e^{-\lambda T_{n}}\left\|\bar{p}\left(T_{n}\right)\right\|_{H}\left\|x\left(T_{n}\right)-\bar{x}\left(T_{n}\right)\right\|_{H}\right] \\
\leq & \lim _{T_{n} \rightarrow \infty}\left(\frac{1}{2} e^{-\lambda T_{n}} \mathbb{E}\left\|\bar{p}\left(T_{n}\right)\right\|_{H}^{2}+\frac{1}{2} e^{-\lambda T_{n}} \mathbb{E}\left\|x\left(T_{n}\right)-\bar{x}\left(T_{n}\right)\right\|_{H}^{2}\right)=0 .
\end{aligned}
$$

Setting $T=T_{n}$ in (3.8) and letting $n \rightarrow \infty$, we obtain

$$
-\mathbb{E}\left[\int_{0}^{\infty} e^{-\lambda s}(\bar{p}(s), h(s)-\bar{h}(s))_{H} d s\right]-\mathbb{E}\left[\int_{0}^{\infty} e^{-\lambda s}(\bar{q}(s), g(s)-\bar{g}(s))_{L_{2}(\Xi, H)} d s\right]
$$




$$
\begin{aligned}
= & \mathbb{E}\left[\int_{0}^{\infty} e^{-\lambda s}\left(x(s)-\bar{x}(s),-\mathcal{H}_{x}(s)-e^{-\lambda \delta} \mathbb{E}\left[\mathcal{H}_{x_{\delta}}(s+\delta) \mid \mathcal{F}_{s}\right]\right)_{H} d s\right] \\
& -\mathbb{E}\left[\int_{0}^{\infty} \lambda e^{-\lambda s}(\bar{p}(s), x(s)-\bar{x}(s))_{H} d s\right] .
\end{aligned}
$$

Then putting (3.9) to (3.6), we can easily obtain the result.

Now, we give the verification theorem of optimality, that is, the sufficient maximum principle for optimal control of Problem 3.2. In addition to Assumption 3.1, verification theorem depends on some convexity assumptions of the Hamiltonian.

Theorem 3.4 (Sufficient maximum principle). Suppose that Assumption 3.1 holds for $\beta<-\left(\gamma+19 M^{2}+1 / 2\right) \wedge 0, \lambda>\left(-\beta+\gamma+\left(3 M / 2+M^{2}\right) \frac{1+e^{2 \beta \delta}}{e^{2 \beta \delta}}\right) \vee(-2 \beta)$. Let $(\bar{u}(\cdot) ; \bar{x}(\cdot))$ be an admissible pair and $(\bar{p}(\cdot), \bar{q}(\cdot))$ be the corresponding adjoint process governed by the adjoint equation (3.5). If the following conditions hold, (i) $\mathcal{H}\left(s, x, x_{\delta}, u_{,} u_{\delta}, \bar{p}, \bar{q}\right)$ is convex in $\left(x, x_{\delta}, u, u_{\delta}\right)$, (ii) $\overline{\mathcal{H}}(s)=\min _{\left(u, u_{\delta}\right) \in \mathcal{U} \times \mathcal{U}} \mathcal{H}\left(s, \bar{x}, \bar{x}(s-\delta), u, u_{\delta}, \bar{p}, \bar{q}\right)$, for almost all $(s, \omega) \in[0, \infty) \times \Omega$, then $(\bar{u}(\cdot) ; \bar{x}(\cdot))$ is an optimal pair of Problem 3.2 .

Proof. Let $(u(\cdot), x(\cdot))$ be an arbitrary admissible pair. According to Lemma 3.3, the difference $J(u(\cdot))-J(\bar{u}(\cdot))$ can be represented by

$$
\begin{aligned}
& J(u(\cdot))-J(\bar{u}(\cdot)) \\
= & \mathbb{E}\left[\int_{0}^{\infty} e^{-\lambda s}\left\{\mathcal{H}(s)-\overline{\mathcal{H}}(s)-\left(\overline{\mathcal{H}}_{x}(s)+e^{-\lambda \delta} \overline{\mathcal{H}}_{x_{\delta}}(s+\delta), x(s)-\bar{x}(s)\right)_{H}\right\} d s\right] .
\end{aligned}
$$

Since for almost all $(s, \omega) \in[0, \infty) \times \Omega, \mathcal{H}\left(s, x, x_{\delta}, u, u_{\delta}, \bar{p}(s), \bar{q}(s)\right)$ is convex in $\left(x, x_{\delta}, u, u_{\delta}\right)$, using Proposition 1.54 of 10 gives

$$
\begin{aligned}
\mathcal{H}(s)-\overline{\mathcal{H}}(s) \geq & \left(\overline{\mathcal{H}}_{x}(s), x(s)-\bar{x}(s)\right)_{H}+\left(\overline{\mathcal{H}}_{x_{\delta}}(s), x(s-\delta)-\bar{x}(s-\delta)\right)_{H} \\
& +\left(\overline{\mathcal{H}}_{u}(s), u(s)-\bar{u}(s)\right)_{U}+\left(\overline{\mathcal{H}}_{u_{\delta}}(s), u(s-\delta)-\bar{u}(s-\delta)\right)_{U} .
\end{aligned}
$$

Moreover, from the optimality condition (ii) and the convex optimization principle (see Proposition 2.21 of 10$]$, for almost all $(s, \omega) \in[0, \infty) \times \Omega$, we obtain

$$
e^{-\lambda s}\left(\overline{\mathcal{H}}_{u}(s), u(s)-\bar{u}(s)\right)_{U}+\left(\overline{\mathcal{H}}_{u_{\delta}}(s), u(s-\delta)-\bar{u}(s-\delta)\right)_{U} \geq 0 .
$$

Plugging (3.11) and 3.12) into (3.10), we obtain

$$
J(u(\cdot))-J(\bar{u}(\cdot)) \geq 0
$$

Thus, $\bar{u}(\cdot)$ is an optimal control process and $(\bar{u}(\cdot) ; \bar{x}(\cdot))$ is an optimal pair. 


\subsection{Necessary maximum principle}

In this subsection, we prove the necessary maximum principle. Suppose that $(\bar{u}(\cdot), \bar{x}(\cdot))$ is an optimal control pair and $(\bar{p}(\cdot), \bar{q}(\cdot))$ is the corresponding adjoint process. Since the control domain $\mathcal{U}$ is convex, for any given control $v(\cdot) \in U_{\text {ad }}$, the perturbed control process $u^{\vartheta}(\cdot):=\bar{u}(\cdot)+\vartheta(v(\cdot)-\bar{u}(\cdot))$ for $0 \leq \vartheta \leq 1$ is also an element of $U_{\text {ad }}$. We denote by $x^{\vartheta}(\cdot)$ the corresponding perturbed state process and assume that $x^{\vartheta}(s)=\varphi(s), s \in[-\delta, 0]$. To simplify our notation, we denote by

$$
\mathcal{H}^{\vartheta}(s):=\mathcal{H}\left(s, x^{\vartheta}(s), x^{\vartheta}(s-\delta), u^{\vartheta}(s), u^{\vartheta}(s-\delta), \bar{p}(s), \bar{q}(s)\right)
$$

Lemma 3.5. Suppose that Assumption 3.1 holds for $\beta<-\left(\gamma+19 M^{2}+1\right) \wedge 0$, then

$$
\mathbb{E}\left[\sup _{s \geq 0} e^{2 \beta s}\left\|x^{\vartheta}(s)-\bar{x}(s)\right\|_{H}^{2}\right]+\mathbb{E}\left[\int_{0}^{\infty} e^{2 \beta s}\left\|x^{\vartheta}(s)-\bar{x}(s)\right\|_{V}^{2} d s\right]=O\left(\vartheta^{2}\right) .
$$

Proof. By Theorem 2.7, we can easily obtain the result.

Then, we get a variational formula of cost functional (3.2).

Lemma 3.6. Suppose that Assumption 3.1 holds for $\beta<-\left(\gamma+19 M^{2}+1\right) \wedge 0, \lambda>$ $\left(-\beta+\gamma+\left(3 M / 2+M^{2}\right) \frac{1+e^{2 \beta \delta}}{e^{2 \beta \delta}}\right) \vee(-2 \beta)$ and for any admissible control $v(\cdot) \in U_{\mathrm{ad}}$, the directional derivative of the cost functional $J(u(\cdot))$ at $\bar{u}(\cdot)$ in the direction $v(\cdot)-\bar{u}(\cdot)$ is given by

$$
\begin{aligned}
& \left.\frac{d}{d \vartheta} J(\bar{u}(\cdot)+\vartheta(v(\cdot)-\bar{u}(\cdot)))\right|_{\vartheta=0} \\
= & \lim _{\vartheta \rightarrow 0} \frac{J(\bar{u}(\cdot)+\vartheta(v(\cdot)-\bar{u}(\cdot)))-J(\bar{u}(\cdot))}{\vartheta} \\
= & \mathbb{E}\left[\int_{0}^{\infty} e^{-\lambda s}\left(\overline{\mathcal{H}}_{u}(s)+e^{-\lambda \delta} \overline{\mathcal{H}}_{u_{\delta}}(s+\delta), v(s)-\bar{u}(s)\right)_{U} d s\right] .
\end{aligned}
$$

Proof. By Lemma 3.3, we have

$$
\begin{aligned}
J\left(u^{\vartheta}(\cdot)\right)-J(\bar{u}(\cdot)) \\
=\mathbb{E}\left[\int _ { 0 } ^ { \infty } e ^ { - \lambda s } \left\{\mathcal{H}^{\vartheta}(s)-\overline{\mathcal{H}}(s)-\left(\overline{\mathcal{H}}_{x}(s)+e^{-\lambda \delta} \overline{\mathcal{H}}_{x_{\delta}}(s+\delta), x^{\vartheta}(s)-\bar{x}(s)\right)_{H}\right.\right. \\
\left.\left.\quad-\left(\overline{\mathcal{H}}_{u}(s)+e^{-\lambda \delta} \overline{\mathcal{H}}_{u_{\delta}}(s+\delta), u^{\vartheta}(s)-\bar{u}(s)\right)_{U}\right\} d s\right] \\
+\mathbb{E}\left[\int_{0}^{\infty} e^{-\lambda s}\left(\overline{\mathcal{H}}_{u}(s)+e^{-\lambda \delta} \overline{\mathcal{H}}_{u_{\delta}}(s+\delta), u^{\vartheta}(s)-\bar{u}(s)\right)_{U} d s\right] .
\end{aligned}
$$

By the expansion of Taylor series and the change of variables, we obtain

$$
\mathbb{E}\left[\int_{0}^{\infty} e^{-\lambda s}\left\{\mathcal{H}^{\vartheta}(s)-\overline{\mathcal{H}}(s)\right\} d s\right]
$$




$$
\begin{aligned}
=\mathbb{E}\left[\int _ { 0 } ^ { \infty } \int _ { 0 } ^ { 1 } e ^ { - \lambda s } \left\{\left(\mathcal{H}_{x}^{\vartheta, \nu}(s), x^{\vartheta}(s)-\bar{x}(s)\right)_{H}+\left(\mathcal{H}_{x_{\delta}, \nu}^{\vartheta}(s), x^{\vartheta}(s-\delta)-\bar{x}(s-\delta)\right)_{H}\right.\right. \\
\left.\left.+\left(\mathcal{H}_{u}^{\vartheta, \nu}(s), u^{\vartheta}(s)-\bar{u}(s)\right)_{U}+\left(\mathcal{H}_{u_{\delta}}^{\vartheta, \nu}(s), u^{\vartheta}(s-\delta)-\bar{u}(s-\delta)\right)_{U}\right\} d \nu d s\right] \\
=\mathbb{E}\left[\int _ { 0 } ^ { \infty } \int _ { 0 } ^ { 1 } e ^ { - \lambda s } \left\{\left(\mathcal{H}_{x}^{\vartheta, \nu}(s)+e^{-\lambda \delta} \mathcal{H}_{x_{\delta}, \nu}^{\vartheta}(s+\delta), x^{\vartheta}(s)-\bar{x}(s)\right)_{H}\right.\right. \\
\left.\left.+\left(\mathcal{H}_{u}^{\vartheta, \nu}(s)+e^{-\lambda \delta} \mathcal{H}_{u_{\delta}}^{\vartheta, \nu}(s+\delta), u^{\vartheta}(s)-\bar{u}(s)\right)_{U}\right\} d \nu d s\right],
\end{aligned}
$$

where

$$
\begin{gathered}
\mathcal{H}^{\vartheta, \nu}(s):=\mathcal{H}\left(s, x^{\vartheta, \nu}(s), x^{\vartheta, \nu}(s-\delta), u^{\vartheta, \nu}(s), u^{\vartheta, \nu}(s-\delta), \bar{p}(s), \bar{q}(s)\right), \\
x^{\vartheta, \nu}(s):=\bar{x}(s)+\nu\left(x^{\vartheta}(s)-\bar{x}(s)\right) \quad \text { and } \quad u^{\vartheta, \nu}(s):=\bar{u}(s)+\nu\left(u^{\vartheta}(s)-\bar{u}(s)\right) .
\end{gathered}
$$

Thus, from (3.15), Lemma 3.5 and the dominated convergence theorem, for $\lambda \geq-2 \beta$, we have

$$
\begin{gathered}
\mathbb{E}\left[\int _ { 0 } ^ { \infty } e ^ { - \lambda s } \left\{\mathcal{H}^{\vartheta}(s)-\overline{\mathcal{H}}(s)-\left(\overline{\mathcal{H}}_{x}(s)+e^{-\lambda \delta} \overline{\mathcal{H}}_{x_{\delta}}(s+\delta), x^{\vartheta}(s)-\bar{x}(s)\right)_{H}\right.\right. \\
\left.\left.-\left(\overline{\mathcal{H}}_{u}(s)+e^{-\lambda \delta} \overline{\mathcal{H}}_{u_{\delta}}(s+\delta), u^{\vartheta}(s)-\bar{u}(s)\right)_{U}\right\} d s\right]=o(\vartheta) .
\end{gathered}
$$

Therefore, putting (3.16) to (3.14), we obtain (3.13).

Theorem 3.7 (Necessary maximum principle). Suppose that Assumption 3.1 holds for $\beta<-\left(\gamma+19 M^{2}+1\right) \wedge 0, \lambda>\left(-\beta+\gamma+\left(3 M / 2+M^{2}\right) \frac{1+e^{2 \beta \delta}}{e^{2 \beta \delta}}\right) \vee(-2 \beta)$. Let $(\bar{u}(\cdot) ; \bar{x}(\cdot))$ be an optimal pair of Problem 3.2 and $(\bar{p}(\cdot), \bar{q}(\cdot))$ be the adjoint process associated with the optimal pair and governed by the adjoint equation (3.5). Then we have the following minimum condition

$$
\left(\overline{\mathcal{H}}_{u}(s)+e^{-\lambda \delta} \mathbb{E}\left[\overline{\mathcal{H}}_{u_{\delta}}(s+\delta) \mid \mathcal{F}_{s}\right], v(s)-\bar{u}(s)\right)_{U} \geq 0
$$

for all $v(\cdot) \in \mathcal{U}$, for a.e. $s \in[0, \infty)$, P-a.s.

Proof. Since $(\bar{u}(\cdot) ; \bar{x}(\cdot))$ is an optimal pair of Problem 3.2 , by Lemma 3.6, we obtain

$$
\begin{aligned}
& \mathbb{E}\left[\int_{0}^{\infty} e^{-\lambda s}\left(\overline{\mathcal{H}}_{u}(s)+e^{-\lambda \delta} \mathbb{E}\left[\overline{\mathcal{H}}_{u_{\delta}}(s+\delta) \mid \mathcal{F}_{s}\right], v(s)-\bar{u}(s)\right)_{U} d s\right] \\
= & \mathbb{E}\left[\int_{0}^{\infty} e^{-\lambda s}\left(\overline{\mathcal{H}}_{u}(s)+e^{-\lambda \delta} \overline{\mathcal{H}}_{u_{\delta}}(s+\delta), v(s)-\bar{u}(s)\right)_{U} d s\right] \\
= & \lim _{\vartheta \rightarrow 0} \frac{J\left(u^{\vartheta}(\cdot)\right)-J(\bar{u}(\cdot))}{\vartheta} \geq 0
\end{aligned}
$$

for any admissible control $v(\cdot) \in U_{\text {ad }}$. Suppose that (3.17) does not hold. Then there exists a $v_{0} \in \mathcal{U}$ such that for some $\delta_{0}>0$ :

$$
e^{-\lambda s}\left(\overline{\mathcal{H}}_{u}(s)+e^{-\lambda \delta} \mathbb{E}\left[\overline{\mathcal{H}}_{u_{\delta}}(s+\delta) \mid \mathcal{F}_{s}\right], v_{0}-\bar{u}(s)\right)_{U} \leq-\delta_{0} .
$$


We define

$$
\begin{gathered}
Q:=\left\{(s, \omega) \in[0, \infty) \times \Omega \mid e^{-\lambda s}\left(\overline{\mathcal{H}}_{u}(s)+e^{-\lambda \delta} \mathbb{E}\left[\overline{\mathcal{H}}_{u_{\delta}}(s+\delta) \mid \mathcal{F}_{s}\right], v_{0}-\bar{u}(s)\right)_{U} \leq-\delta_{0}\right\}, \\
Q_{s}:=\{\omega \in \Omega \mid(s, \omega) \in Q\},
\end{gathered}
$$

and an admissible control by

$$
v(s)= \begin{cases}v_{0}, & (s, \omega) \in Q \\ \bar{u}(s), & \text { otherwise }\end{cases}
$$

Therefore

$$
\begin{aligned}
0 & \leq \mathbb{E}\left[\int_{0}^{\infty} e^{-\lambda s}\left(\overline{\mathcal{H}}_{u}(s)+e^{-\lambda \delta} \mathbb{E}\left[\overline{\mathcal{H}}_{u_{\delta}}(s+\delta) \mid \mathcal{F}_{s}\right], v(s)-\bar{u}(s)\right)_{U} d s\right] \\
& \leq-\delta_{0} \int_{0}^{\infty} P\left(Q_{s}\right) d s<0 .
\end{aligned}
$$

This leads to a contradiction, thus the inequality 3.17 holds.

Remark 3.8. The main contribution of this section is to find the appropriate adjoint equation (3.5). By this adjoint equation, the sufficient maximum principle and necessary maximum principle of optimal control problem are obtained. We note that the adjoint equation (3.5) is different from the adjoint equation in finite horizon case (see the adjoint equation (33) in 17$]$ ). Specifically, the anticipated term $\mathbb{E}\left[\mathcal{H}_{x_{\delta}}(s+\delta) \mid \mathcal{F}_{s}\right]$ is multiplied by $e^{-\lambda \delta}$. Therefore, the necessary condition 3.17 in Theorem 3.7 is different from the necessary condition in finite horizon case (see Theorem 4.2 in 17]). Moreover, in proving Theorem 3.4, equality 3.10 in Lemma 3.3 is used, which is also different from equality (38) in $[17$.

\section{Example}

As an application of our results, we consider the stochastic delay partial differential equation (see also Subsection 6.1 of [17] for finite horizon case)

$$
\begin{aligned}
d y(t, z)= & \left\{\partial_{z^{i}}\left[a^{i j}(t, z) \partial_{z^{j}} y(t, z)\right]+b^{i}(t, z) \partial_{z^{i}} y(t, z)+c(t, z) y(t, z)\right. \\
& +h(t, z, y(t, z), y(t-\delta, z), u(t, z), u(t-\delta, z))\} d t \\
& +g(t, z, y(t, z), y(t-\delta, z), u(t, z), u(t-\delta, z)) d W(t), \quad(t, z) \in[0, \infty) \times G, \\
y(t, z)= & \xi_{0}(t, z), \quad(t, z) \in[-\delta, 0] \times G, \\
y(t, z)= & 0, \quad(t, z) \in[-\delta, \infty) \times \partial G, \\
u(t, z)= & u_{0}(t, z), \quad(t, z) \in[-\delta, 0] \times G,
\end{aligned}
$$


where $G$ is a bounded, open set in $\mathbb{R}^{d}$ with boundary $\Gamma$, which is $C^{\infty}$-manifold of dimension of $d-1 ; u(t, z)$ is the control process valued in a convex subset $\mathfrak{U}$ of $\mathbb{R}$, and the Einstein summation convention applies to $\partial_{z^{i}}\left[a^{i j}(t, z) \partial_{z^{j}} y(t, z)\right]$ and $b^{i}(t, z) \partial_{z^{i}} y(t, z)$. Here the coefficients $a^{i j}, b^{i}, c:[0, \infty) \times \Omega \times G \rightarrow \mathbb{R}, h, g:[0, \infty) \times \Omega \times G \times \mathbb{R} \times \mathbb{R} \times \mathfrak{U} \times \mathfrak{U} \rightarrow \mathbb{R}$, $\xi_{0}:[-\delta, 0] \times \Omega \times G \rightarrow \mathbb{R}$ and $u_{0}:[-\delta, 0] \times \Omega \times G \rightarrow \mathfrak{U}$, for all $i, j=1,2, \ldots, d$, are given random mappings and satisfy the suitable measurability.

Definition 4.1. A control process $u(\cdot, \cdot)$ is said to be admissible if $u(\cdot, \cdot) \in L_{\mathcal{P}}^{2}(\Omega$; $\left.L_{\beta}^{2}\left(L^{2}(G)\right)\right)$ for some $\beta$ which will be given later and $u(t, z) \in \mathfrak{U}$, a.e. $(t, z) \in[0, \infty) \times G$, $P$-a.s. Write $U_{\text {ad }}$ for the set of all admissible control processes.

We introduce the cost functional

$$
J(u(\cdot, \cdot))=\mathbb{E} \int_{0}^{\infty} e^{-\lambda t} \int_{G} k(t, z, y(t, z), y(t-\delta, z), u(t, z), u(t-\delta, z)) d z d t .
$$

To make the control problem well-defined, we give the following assumptions on coefficients:

Assumption 4.2. (i) The functions $\left(a^{i j}\right)_{d \times d},\left(b^{i}\right)_{d}$ and c are $\mathcal{P} \times \mathcal{B}(G)$-measurable with values in the set of real symmetric $d \times d$ matrices, $\mathbb{R}^{d}$ and $\mathbb{R}$ respectively, and are bounded by $K$.

(ii) The super-parabolic condition holds, i.e.,

$$
\kappa I \leq 2\left(a^{i j}\right)_{d \times d}(t, \omega, z) \leq K I, \quad \forall(t, \omega, z) \in[0, \infty) \times \Omega \times G,
$$

where $I$ is the $(d \times d)$-identity matrix and $\kappa>0$.

(iii) $u_{0}(\cdot, \cdot) \in L_{\mathcal{P}}^{2}\left([0,-\delta] \times \Omega ; L_{\beta}^{2}\left(L^{2}(G)\right)\right)$ and $u_{0}(t, z) \in \mathfrak{U}, \xi_{0}(\cdot, \cdot) \in L_{\mathcal{P}}^{2}(\Omega ; C[-\delta, 0]$; $\left.L^{2}(G)\right)$, and $h(\cdot, \cdot, 0,0,0,0), g(\cdot, \cdot, 0,0,0,0) \in L_{\mathcal{P}}^{2}\left(\Omega ; L_{\beta}^{2}\left(L^{2}(G)\right)\right) ;$

(iv) for almost all $(t, \omega, z) \in[0, \infty) \times \Omega \times G, h$ and $g$ are differentiable with respect to $\left(y, y_{\delta}, u, u_{\delta}\right) \in \mathbb{R} \times \mathbb{R} \times \mathfrak{U} \times \mathfrak{U}$ with continuous and uniformly bounded derivatives $h_{y}$, $h_{y_{\delta}}, g_{y}, g_{y_{\delta}}, h_{u}, h_{u_{\delta}}, g_{u}, g_{u_{\delta}}$ i.e., there exists a constant $M>0$ such that

$$
\sup _{\left(s, \omega, y, y_{\delta}, u, u_{\delta}\right) \in[0, \infty) \times \Omega \times H \times H \times \mathfrak{U} \times \mathfrak{U}}\left\|h_{y}\left(s, y, y_{\delta}, u, u_{\delta}\right)\right\|_{L(H)} \leq M,
$$

and $h_{y_{\delta}}, g_{y}, g_{y_{\delta}}, h_{u}, h_{u_{\delta}}, g_{u}, g_{u_{\delta}}$ are similar to the above conditions;

(v) for almost all $(t, \omega, z) \in[0, \infty) \times \Omega \times G, k$ is differentiable with respect to $\left(y, y_{\delta}, u, u_{\delta}\right)$ with continuous derivatives $k_{y}, k_{y_{\delta}}, k_{u}, k_{u_{\delta}}$; moreover, for almost all $(t, \omega) \in[0, \infty) \times$ 
$\Omega$, there exists a constant $C>0$ such that for all $\left(y, y_{\delta}, u, u_{\delta}\right) \in \mathbb{R} \times \mathbb{R} \times \mathfrak{U} \times \mathfrak{U}$,

$$
\begin{aligned}
& \left|k\left(t, z, y, y_{\delta}, u, u_{\delta}\right)\right| \leq C\left(1+|y|^{2}+\left|y_{\delta}\right|^{2}+|u|^{2}+\left|u_{\delta}\right|^{2}\right) \\
& \quad\left|k_{y}\left(t, z, y, y_{\delta}, u, u_{\delta}\right)\right|+\left|k_{y_{\delta}}\left(t, z, y, y_{\delta}, u, u_{\delta}\right)\right| \\
& \quad+\left|k_{u}\left(t, z, y, y_{\delta}, u, u_{\delta}\right)\right|+\left|k_{u_{\delta}}\left(t, z, y, y_{\delta}, u, u_{\delta}\right)\right| \\
& \leq C\left(1+|y|+\left|y_{\delta}\right|+|u|+\left|u_{\delta}\right|\right) .
\end{aligned}
$$

To apply our abstract theoretical results in Section 3 , we set $V=H_{0}^{1}(G), H=L^{2}(G)$, $V^{*}=H_{0}^{-1}(G), U=L^{2}(G)$ and $\mathcal{U}=\{u(\cdot) \mid u(\cdot) \in U, u(z) \in \mathfrak{U}\}$. Hence $\left(V, H, V^{*}\right)$ is a Gelfand triple. Next we define the second-order differential operators $A$ and the nonlinear operators in the control system as

$$
A(t) \phi(z) \triangleq-\partial_{z^{i}}\left[a^{i j}(t, z) \partial_{z^{j}} \phi(z)\right]-b^{i}(t, z) \partial_{z^{i}} \phi(z)-c(t, z) \phi(z), \quad \forall \phi \in V
$$

and

$$
\begin{aligned}
h\left(t, \phi, \phi_{\delta}, u, u_{\delta}\right)(z) & \triangleq h(t, z, \phi(t, z), \phi(t-\delta, z), u(t, z), u(t-\delta, z)), \\
g\left(t, \phi, \phi_{\delta}, u, u_{\delta}\right)(z) & \triangleq g(t, z, \phi(t, z), \phi(t-\delta, z), u(t, z), u(t-\delta, z)), \\
k\left(t, \phi, \phi_{\delta}, u, u_{\delta}\right) & \triangleq \int_{G} k(t, z, \phi(t, z), \phi(t-\delta, z), u(t, z), u(t-\delta, z)) d z, \quad \forall \phi \in H .
\end{aligned}
$$

Note that the adjoint operator of $A$ is

$$
A^{*}(t) \phi(z) \triangleq-\partial_{z^{i}}\left[a^{i j}(t, z) \partial_{z^{j}} \phi(z)\right]+b^{i}(t, z) \partial_{z^{i}} \phi(z)-\left[c(t, z)-\partial_{z^{i}} b^{i}(t, z)\right] \phi(z), \quad \forall \phi \in V .
$$

Then we can rewrite the state equation (4.1) as an abstract SDEE in the Gelfand triple $\left(V, H, V^{*}\right)$ :

$$
\begin{aligned}
d y(t)= & {[-A(t) y(t)+h(t, y(t), y(t-\delta), u(t), u(t-\delta))] d t } \\
& +g(t, y(t), y(t-\delta), u(t), u(t-\delta)) d W(t), \quad t \in[0, \infty) \\
y(t)= & \xi_{0}(t), \quad t \in[-\delta, 0], \\
u(t)= & u_{0}(t), \quad t \in[-\delta, 0],
\end{aligned}
$$

and the cost functional 4.2 as

$$
J(u(\cdot))=\mathbb{E}\left[\int_{0}^{\infty} e^{-\lambda t} k(t, y(t), y(t-\delta), u(t), u(t-\delta)) d t\right] .
$$

The corresponding optimal control problem is formulated as follows:

Problem 4.3. Find an admissible control $\bar{u}(\cdot)$ such that

$$
J(\bar{u}(\cdot))=\inf _{u(\cdot) \in U_{\mathrm{ad}}} J(u(\cdot))
$$


By Assumption 4.2(i), (ii), the operator $A$ satisfies the coercivity and boundedness conditions, i.e., (ii) and (iii) in Assumption 2.2. The conditions (i), (iii) and (iv) in Assumption 3.1 can be obtained from (iii), (iv) and (v) in Assumption 4.2, respectively. Therefore, under Assumption 4.2, the coefficients $(A, h, g, k)$ satisfy Assumption 3.1. So for any admissible control $u(\cdot), \beta<-\left(\gamma+19 M^{2}+1 / 2\right) \wedge 0$ and $\lambda \geq-2 \beta$, the state equation has a unique solution $y(\cdot)$ and the corresponding optimal control problem is well-defined according to Theorem 2.5 .

The corresponding Hamiltonian $\mathcal{H}:[0, \infty) \times \Omega \times H \times H \times \mathcal{U} \times \mathcal{U} \times V \times H \rightarrow \mathbb{R}$ is given by

$$
\mathcal{H}\left(t, y, y_{\delta}, u, u_{\delta}, p, q\right) \triangleq\left(h\left(t, y, y_{\delta}, u, u_{\delta}\right), p\right)_{H}+\left(g\left(t, y, y_{\delta}, u, u_{\delta}\right), q\right)_{H}+k\left(t, y, y_{\delta}, u, u_{\delta}\right)
$$

Then the adjoint equation is governed by

$$
d p(s)=\left\{A^{*}(s) p(s)-\mathcal{H}_{y}(s)-e^{-\lambda \delta} \mathbb{E}\left[\mathcal{H}_{y_{\delta}}(s+\delta) \mid \mathcal{F}_{s}\right]\right\} d s+q(s) d W(s), \quad s \in[0, \infty)
$$

where we denote by

$$
\mathcal{H}(t) \triangleq \mathcal{H}(t, y(t), y(t-\delta), u(t), u(t-\delta), p(t), q(t))
$$

Under Assumptions 4.2, the adjoint equation (4.3) admits a unique solution $(p(\cdot), q(\cdot)) \in$ $L_{\mathcal{P}}^{2}\left(\Omega ; L_{\beta}^{2}(H)\right) \times L_{\mathcal{P}}^{2}\left(\Omega ; L_{\beta}^{2}\left(L_{2}(\Xi, H)\right)\right)$ for $\lambda>\left(-\beta+\gamma+\left(3 M / 2+M^{2}\right) \frac{1+e^{2 \beta \delta}}{e^{2 \beta \delta}}\right) \vee 0$ according to Theorem 2.13. Then, for $\beta<-\left(\gamma+19 M^{2}+1 / 2\right) \wedge 0$ and $\lambda>(-\beta+\gamma+(3 M / 2+$ $\left.\left.M^{2}\right) \frac{1+e^{2 \beta \delta}}{e^{2 \beta \delta}}\right) \vee(-2 \beta)$, we can apply the abstract result Theorem 3.7 directly in this case. Consequently, we write the following necessary condition for the optimal control:

$$
\int_{G}\left[\left(\overline{\mathcal{H}}_{u}(t)+e^{-\lambda \delta} \mathbb{E}\left[\overline{\mathcal{H}}_{u_{\delta}}(t+\delta) \mid \mathcal{F}_{t}\right]\right)(v(z)-\bar{u}(t, z))\right] d z \geq 0
$$

for all $v \in \mathcal{U}$, for a.e. $t \in[0, \infty)$, P-a.s. Moreover, if $\mathcal{H}\left(t, y, y_{\delta}, u, u_{\delta}, p, q\right)$ is convex in $\left(y, y_{\delta}, u, u_{\delta}\right)$, the sufficient maximum principle also hold for this case according to Theorem 3.4 .

\section{References}

[1] N. Agram, S. Haadem, B. Øksendal and F. Proske, A maximum principle for infinite horizon delay equations, SIAM J. Math. Anal. 45 (2013), no. 4, 2499-2522.

[2] N. Agram and B. Øksendal, Infinite horizon optimal control of forward-backward stochastic differential equations with delay, J. Comput. Appl. Math. 259 (2014), part B, 336-349. 
[3] T. Caraballo, M. J. Garrido-Atienza and J. Real, Existence and uniqueness of solutions for delay stochastic evolution equations, Stochastic Anal. Appl. 20 (2002), no. 6, 1225-1256.

[4] M.-H. Chang, Stochastic Control of Hereditary Systems and Applications, Stochastic Modelling and Applied Probability 59, Springer, New York, 2008.

[5] L. Chen and Z. Wu, Maximum principle for the stochastic optimal control problem with delay and application, Automatica J. IFAC 46 (2010), no. 6, 1074-1080.

[6] G. Da Prato and J. Zabczyk, Stochastic Equations in Infinite Dimensions, Encyclopedia of Mathematics and its Applications 44, Cambidge University Press, Cambidge, 1992.

[7] H. Dai, J. Zhou and H. Li, Infinite horizon stochastic maximum principle for stochastic delay evolution equations in Hilbert spaces, Bull. Malays. Math. Sci. Soc. 44 (2021), no. 5, 3229-3258.

[8] D. David, Optimal control of stochastic delayed systems with jumps, available at https://hal.archives-ouvertes.fr/hal-00373642/document, (2008), 32 pp.

[9] B. Diomande, Maximum principle for stochastic delay differential equation in infinite dimension spaces, available at https://www.minet.uni-jena.de/Marie-CurieITN/SMIF/talks/Diomande.pdf, (2011).

[10] I. Ekeland and R. Temam, Convex Analysis and Variational Problems, Studies in Mathematics and its Applications 1, North-Holland, Amsterdam-Oxford, 1976.

[11] I. Elsanosi, B. Øksendal and A. Sulem, Some solvable stochastic control problems with delay, Stochastics Stochastics Rep. 71 (2000), no. 1-2, 69-89.

[12] S. Federico and P. Tankov, Finite-dimensional representations for controlled diffusions with delay, Appl. Math. Optim. 71 (2015), no. 1, 165-194.

[13] W. H. Fleming and H. M. Soner, Controlled Markov Processes and Viscosity Solutions, Applications of Mathematics (New York) 25, Springer-Verlag, New York, 1993.

[14] M. Fuhrman and G. Tessitore, Infinite horizon backward stochastic differential equations and elliptic equations in Hilbert spaces, Ann. Probab. 32 (2004), no. 1B, 607660 .

[15] N. V. Krylov and B. L. Rozovskii, Stochastic evolution equations, J. Sov. Math. 16 (1981), no. 4, 1233-1277. 
[16] Q. Meng and Y. Shen, Optimal control of mean-field jump-diffusion systems with delay: A stochastic maximum principle approach, J. Comput. Appl. Math. 279 (2015), 13-30.

[17] _ Optimal control for stochastic delay evolution equations, Appl. Math. Optim. 74 (2016), no. 1, 53-89.

[18] S. E. A. Mohammed, Stochastic Functional Differential Equations, Research Notes in Mathematics 99, Pitman, Boston, MA, 1984.

[19] B. Øksendal and A. Sulem, A maximum principle for optimal control of stochastic systems with delay, with applications to finance, in: Optimal Control and Partial Differential Equations, 64-79, IOS, Amsterdam, 2000.

[20] B. Øksendal, A. Sulem and T. Zhang, Optimal control of stochastic delay equations and time-advanced backward stochastic differential equations, Adv. in Appl. Probab. 43 (2011), no. 2, 572-596.

[21] S. Peng and Z. Yang, Anticipated backward stochastic differential equations, Ann. Probab. 37 (2009), no. 3, 877-902.

[22] Y. Shen, Q. Meng and P. Shi, Maximum principle for mean-field jump-diffusion stochastic delay differential equations and its application to finance, Automatica J. IFAC 50 (2014), no. 6, 1565-1579.

[23] J. Yong and X. Y. Zhou, Stochastic Controls: Hamiltonian systems and HJB equations, Applications of Mathematics (New York) 43, Springer-Verlag, New York, 1999.

Han Li, Jianjun Zhou, Haoran Dai, Biteng Xu and Wenxu Dong

College of Science, Northwest A\&F University, Yangling, Shaanxi 712100, China

E-mail addresses: lihan199501@163.com, zhoujianjun@nwsuaf.edu.cn,

15615350357@163.com, bitengxu@163.com, 501498413@qq.com 